Revista Brasileira de Cartografia

ISSN 1808-0936 | https://doi.org/10.14393/revbrascartogr

Sociedade Brasileira de Cartografia, Geodésia, Fotogrametria e Sensoriamento Remoto

\title{
Estado da Arte do Sensoriamento Remoto de Radar: Fundamentos, Sensores, Processamento de Imagens e Aplicações
}

\author{
State-of-the-art of the Radar Remote Sensing: Fundamentals, Sensors, Image Processing, \\ and Applications
}

Edson Eyji Sano ${ }^{1}$, Eraldo Aparecido Trondoli Matricardi ${ }^{2}$ e Flávio Fortes Camargo ${ }^{3}$

1 Embrapa Cerrados, Planaltina, Brasil. edson.sano@embrapa.br

ORCID: https://orcid.org/0000-0001-5760-556X

2 Universidade de Brasília, Departamento de Engenharia Florestal, Brasília, Brasil. E-mail. ematricardi @unb.br ORCID: https://orcid.org/0000-0002-5323-6100

3 Departamento Nacional de Infraestrutura de Transportes, Brasília, Brasil. E-mail. flavio.camargo@dnit.gov.br

ORCID: https://orcid.org/0000-0002-7815-7919

Resumo: Este artigo aborda o estado da arte do sensoriamento remoto por radar e foi elaborado para fazer parte da edição especial de comemoração dos 50 anos desta revista. Neste estudo, é apresentada uma breve introdução sobre os fundamentos do sensoriamento remoto por radar, com destaque para os parâmetros mais importantes de imageamento e da superfície terrestre envolvidos no processo de obtenção de imagens de radar. Ênfase é dada para o comprimento de onda, polarização das ondas eletromagnéticas e geometria de obtenção de imagens (parâmetros de imageamento) e para a umidade de solos e da vegetação, rugosidade do terreno e estrutura da vegetação (parâmetros da superfície terrestre). Em seguida, são apresentados os principais sensores orbitais de radar de abertura sintética que estão atualmente em operação e os principais processamentos digitais de imagens de radar, destacando-se a conversão dos valores digitais para coeficientes de retroespalhamento, os filtros espaciais para redução do ruído speckle, as técnicas de decomposição de imagens e o processamento InSAR. Finalmente, é apresentada uma breve discussão sobre algumas aplicações potenciais, com especial atenção para o monitoramento de derrame de óleo em plataformas continentais, estimativa de biomassa aérea, monitoramento de desmatamento em coberturas florestais tropicais, detecção de áreas de plantio de arroz irrigado e estimativa de umidade de solos.

Palavras-chave: SAR. Sensoriamento remoto. Retroespalhamento. Satélites de recursos terrestres.

\begin{abstract}
This article discusses the state-of-the-art of radar remote sensing and was prepared as part of the special edition of the 50th anniversary of this journal. This study briefly introduces the fundamentals of radar remote sensing, highlighting the most important aspects of image acquisition modes and biophysical parameters of the Earth's surface involved in the process of acquiring radar images. We emphasized wavelength, polarization of electromagnetic waves, image acquisition geometry (imaging parameters), soil and vegetation water content, terrain roughness, and vegetation structure (biophysical parameters of the Earth's surface). Subsequently, the main orbital synthetic aperture radar sensors currently in operation and the main options of radar image processing are presented, highlighting the conversion of digital values to backscatter coefficients, spatial filters to reduce speckle noise, image decomposition techniques, and InSAR processing. Finally, we briefly discuss some potential applications, with special attention to the monitoring of oil spills in continental platforms, above-ground biomass estimation, deforestation monitoring in tropical forests, detection of irrigated rice cropping areas, and soil moisture estimation.
\end{abstract}

Keywords: SAR. Remote sensing. Backscattering. Earth resources satellites. 


\section{INTRODUÇÃO}

O sensoriamento remoto por radar em larga escala foi usado pela primeira vez no Brasil pelo projeto RADAMBRASIL durante a década de 1970 para mapear recursos naturais do Brasil. Esse projeto foi inicialmente planejado para cobrir a Amazônia brasileira, porém, devido ao seu sucesso, foi estendido para todo o país em 1975. Nesse projeto, foram obtidas imagens de radar em formato analógico pelo avião norteamericano Caravelle. O sensor transportado por essa aeronave consistia em um sistema de radar de visada lateral denominado GEMS (Goodyear Mapping System 1000), o qual operou em comprimento de onda de 3,2 cm (banda X), polarização $\mathrm{HH}$, faixa de imageamento de $37 \mathrm{~km}$, direção de voo norte-sul e sobreposição lateral de 25\% (LIMA, 2014). Essas imagens foram disponibilizadas em papel fotográfico na escala 1:250.000 e grade regular de $1^{\circ} 00^{\prime}$ x $1^{\circ} 30^{\prime}$. A escolha de um sistema de radar foi baseada na sua capacidade de obter imagens independentemente da cobertura de nuvens e das condições de iluminação solar. Especialistas foram contratados pelo Ministério de Minas e Energia para interpretar visualmente os mosaicos semicontrolados impressos em tamanho A0 para produzir mapas de solo, geomorfologia, geologia e vegetação, entre outros mapas temáticos. Os relatórios estão disponíveis gratuitamente para download na biblioteca do Instituto Brasileiro de Geografia e Estatística (IBGE) (IBGE, 2020).

O primeiro sistema orbital de radar de abertura sintética (SAR) foi lançado em 1978 a bordo do satélite SEASAT pela agência espacial norte-americana (NASA). Esse sistema foi projetado principalmente para o monitoramento global de fenômenos oceanográficos e para auxiliar na determinação dos principais requisitos necessários para o estudo de oceanos via sensoriamento remoto orbital (EVANS et al., 2005). Os objetivos específicos incluíram a coleta de dados de vento e temperatura da superfície do mar, altura das ondas, vapor d'água na atmosfera e propriedades dos gelos presentes no mar, entre outros fenômenos. Em 1981, 1984 e 1994, foi lançada a série Shuttle Imaging Radar (SIR), denominada de SIR-A, SIR-B e SIR-C/X-SAR, respectivamente (CIMINO; ELACHI; SETTLE, 1986; EVANS, 2006). O legado científico dessas missões a bordo de ônibus espaciais é extenso, notadamente nas áreas de estimativa de biomassa, umidade do solo e monitoramento da floresta amazônica (STONE; WOODWELL, 1988; HESS et al., 1995; FERRAZOLI et al., 1997; SAATCHI; SOARES; ALVES, 1997; ALSDORF; SMITH; MELACK, 2001).

Graças ao sucesso dessas missões baseadas em ônibus espaciais, três sistemas de satélite SAR foram lançados pela Agência Espacial Europeia (ESA), Agência Espacial Canadense (CSA) e Agência de Exploração Aeroespacial do Japão (JAXA): o Satélite de Recursos Terrestres (ERS-1), o RADARSAT-1 e o Satélite Japonês de Recursos Terrestres-1 (JERS-1), respectivamente. Esses satélites operavam em frequência e polarização única devido ao grande volume de dados que eram gerados. Vários pesquisadores analisaram o potencial desses dados SAR para estimar diferentes parâmetros biofísicos (DOBSON; PIERCE; ULABY, 1996; SANO et al., 1998a; SRIVASTAVA et al., 2003). No entanto, houve uma intensa demanda da comunidade científica pela polarização múltipla para melhorar o potencial de discriminação e mapeamento de alvos específicos de interesse, por exemplo, umidade de solos, minimizando a influência de outros parâmetros biofísicos como rugosidade do solo, estrutura da vegetação e biomassa. A capacidade de polarização dupla ou quádrupla só foi disponibilizada na última geração de satélites de radar, lançados após 2005.

A principal vantagem dos sensores de radar é a sua capacidade de obter imagens independentemente das condições de cobertura de nuvens (HENDERSON; LEWIS, 1998; SANO; MENESES; ALMEIDA, 2019). Isso ocorre porque os comprimentos de onda das bandas espectrais dos sensores SAR são muito maiores do que o diâmetro médio das partículas e moléculas encontradas na atmosfera. Como resultado, os sistemas SAR são mais adequados do que os sensores ópticos para monitorar a dinâmica ambiental de países tropicais da América do Sul (por exemplo, Brasil, Guiana e Peru), África Central (Congo, República Democrática do Congo e Gabão) e Sudeste Asiático (Indonésia, Malásia e Cingapura). Nessas regiões, predominam as condições persistentes de cobertura de nuvens ao longo do ano. Outra vantagem dos dados SAR é a sua independência das condições de iluminação solar. As seguintes tendências no campo do sensoriamento remoto por radar podem ser destacadas: disponibilidade crescente de dados gratuitos (até o momento, apenas dados de um único satélite de radar estão sendo disponibilizados gratuitamente na internet); disponibilidade crescente de plataformas de processamento e armazenamento em nuvem, como os disponibilizados pelo Google ${ }^{\mathrm{TM}} \mathrm{e}$ Amazon $^{\mathrm{TM}}$ para acessar e processar grandes volumes de dados; continuidade dos programas SAR existentes e 
lançamento de novos programas, garantindo a construção de séries temporais longas e consistentes de dados de radar; e disponibilidade de produtos SAR prontos para serem analisados, reduzindo mão-de-obra e tempo de processamento.

Este artigo de revisão objetiva apresentar o estado da arte do sensoriamento remoto por radar e foi elaborado para fazer parte da edição especial de 50 anos desta revista científica. Na Seção 2, são discutidos os fundamentos do sensoriamento remoto por radar. Nas Seções 3 e 4, são apresentados os sistemas SAR atualmente em operação e os sensores de radar a bordo da aeronave R99B da Força Aérea Brasileira (FAB), respectivamente. Na Seção 5, são mostrados alguns exemplos de aplicações. As considerações finais são apresentadas na Seção 6.

\section{FUNDAMENTOS DE SENSORIAMENTO REMOTO POR RADAR}

O SAR é um sistema ativo que opera na região espectral de micro-ondas, cujo comprimento de onda varia aproximadamente de $0,8 \mathrm{~cm}$ a $120 \mathrm{~cm}$ (Figura 1). Os sistemas ativos usam sua própria fonte de radiação eletromagnética (REM), geralmente uma antena que transmite pulsos de energia e registra o componente retroespalhado da radiação emitida. Retroespalhamento é um termo usado para sensores ativos e é equivalente à energia solar refletida e detectada por sensores passivos. As bandas espectrais dos sistemas SAR são especificadas pelos seguintes códigos: $\mathrm{K}(0,8 \mathrm{~cm}-2,5 \mathrm{~cm}) ; \mathrm{X}(2,5 \mathrm{~cm}-3,8 \mathrm{~cm}) ; \mathrm{C}(3,8 \mathrm{~cm}-7,5 \mathrm{~cm}) ; \mathrm{S}(7,5$ $\mathrm{cm}-15 \mathrm{~cm}) ; \mathrm{L}(15 \mathrm{~cm}-60 \mathrm{~cm}) ;$ e $\mathrm{P}(60 \mathrm{~cm}-120 \mathrm{~cm})$. Não há significado prático para cada código descrito por estas letras maiúsculas. Os desenvolvedores usaram descritores alfabéticos comuns e aparentemente aleatórios para comprimentos de onda ou frequências durante a Segunda Guerra Mundial (JENSEN, 2000). Os intervalos de frequência correspondentes também podem ser usados para especificar bandas espectrais. Normalmente, os engenheiros optam por frequência, pois quando a radiação atravessa materiais com densidades diferentes, a frequência permanece constante, enquanto os pesquisadores de aplicação preferem comprimentos de onda. Neste estudo, o comprimento de onda foi escolhido para designar as bandas espectrais de diferentes sensores. Atualmente, apenas as bandas X, C e L são usadas nas plataformas orbitais, não havendo sistemas orbitais operando com duas ou três bandas diferentes. Quanto maior o comprimento de onda, maior é a capacidade de penetração dos sinais emitidos nas copas da vegetação ou nas superfícies do solo. Em solos secos, a capacidade de penetração dos sinais de radar é, no máximo, a metade de seu comprimento de onda. No dossel da vegetação, depende do nível de biomassa e da estrutura da vegetação. Portanto, a seleção do melhor comprimento de onda dependerá do tipo de aplicação ou do objetivo do estudo.

Figura 1 - Designação das bandas de acordo com o comprimento de onda e frequência dos sistemas SAR usados para coletar informações a partir de plataformas aéreas ou orbitais.

Comprimento de onda $(\mathrm{cm})$

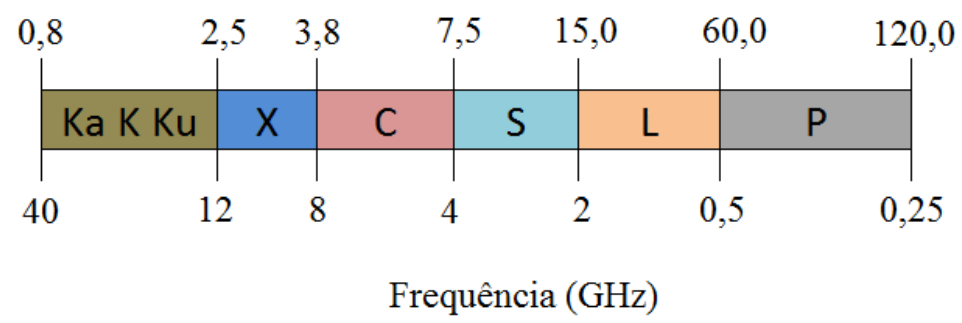

Fonte: Modificada de Ouchi (2013).

Outro parâmetro de imageamento importante dos sistemas SAR é a polarização das energias emitidas e retroespalhadas. Por serem sensores ativos, os sistemas SAR podem configurar a antena para transmitir e receber componentes elétricos de sinais em um único plano perpendicular à direção de propagação. Normalmente, os sistemas de radar são projetados para operar nos planos horizontal (H) e vertical (V). Como um sinal transmitido despolariza ao atingir o terreno, um determinado sensor pode operar em quatro combinações de polarização diferentes, $\mathrm{HH}, \mathrm{HV}, \mathrm{VH}$ e VV. A primeira letra se refere ao sinal transmitido, enquanto a segunda letra se refere ao sinal retroespalhado. Os sinais $\mathrm{HH}$ e VV são conhecidos como 
polarização paralela, enquanto os sinais HV e VH são conhecidos como polarização cruzada. Normalmente, a intensidade dos sinais retroespalhados e relacionados com polarização paralela é maior do que a dos relacionados com polarização cruzada. Os sistemas de radar podem ter uma, duas ou todas as quatro combinações de polarização de transmissão e recepção. Sempre que um sensor SAR opera com capacidade de polarização quádrupla, há a oportunidade de gerar composições coloridas RGB de imagens nas polarizações $\mathrm{HH}, \mathrm{HV}$ e VH ou VV. Quando o sistema opera com duas polarizações, pode-se gerar uma terceira imagem aplicando qualquer operação matemática, como adição, subtração, divisão, multiplicação ou média.

Quando uma antena emite um pulso de energia em direção ao terreno, é necessário considerar as seguintes direções e ângulos: direção azimutal e direção de alcance; e ângulo de incidência ou ângulo de depressão. A direção azimutal refere-se à direção do movimento de uma plataforma (aeronave ou satélite) em linha reta. Pulsos de energia na faixa espectral de micro-ondas são transmitidos para o terreno perpendicularmente à direção azimutal, isto é, a direção de alcance. Algumas feições lineares do terreno como estruturas geológicas relacionadas com falhas e lineamentos e localizadas perpendicularmente à direção de alcance, geralmente são mais bem realçadas em relação às mesmas feições localizadas paralelamente à direção do alcance. O ângulo de depressão $(\gamma)$ é o ângulo formado entre o plano horizontal e o pulso de energia emitido pela antena em direção ao terreno, enquanto o ângulo de incidência $(\theta)$ é o ângulo formado entre a linha perpendicular ao terreno e o pulso de energia emitido por a antena. Quando o terreno é plano, o ângulo de depressão e o ângulo de incidência são complementares.

De acordo com o modelo corpuscular de REM $(\mathrm{E}=\mathrm{hc} / \lambda$, onde $\mathrm{E}=$ energia; $\mathrm{h}=$ constante de Planck; e $\lambda=$ comprimento de onda), a energia na faixa espectral de micro-ondas é insuficiente para interagir com os átomos e moléculas dos alvos, não sendo possível extrair informações sobre sua composição química, que é a base do sensoriamento remoto óptico. Assim, a extração de informações de imagens de radar é baseada nas propriedades geométricas e dielétricas dos materiais (PARADELLA et al., 2005). Em outras palavras, a umidade do solo, a rugosidade do terreno, o conteúdo de água da vegetação e a estrutura da vegetação desempenham papel fundamental na análise de imagens de radar. Quando um solo seco é umedecido, pode-se perceber visualmente que o solo torna-se mais escuro devido ao aumento da absorção da REM no intervalo visível do espectro eletromagnético. O mesmo solo se torna mais claro nas imagens SAR porque ocorre um aumento na constante dielétrica $(\varepsilon)$ do solo. Solos secos apresentam valores baixos de constante dielétrica (3 $<\varepsilon<8$ ), enquanto a $\varepsilon$ de água líquida varia de 48 a 80, dependendo da sua temperatura (SANO; MENESES; ALMEIDA, 2019). Portanto, quando o teor de água do solo aumenta, $\varepsilon$ aumenta e, consequentemente, a energia retroespalhada é mais intensa. O mesmo fenômeno ocorre quando há aumento no teor de água das folhas verdes. Essas mudanças e efeitos no solo e na vegetação são a base para o uso de dados de radar para estimar teor de umidade do solo ou discriminar fitofisionomias.

A rugosidade do terreno também desempenha um papel importante no processo de retroespalhamento, uma vez que a magnitude da rugosidade do solo é aproximadamente a mesma magnitude dos comprimentos de onda na faixa de micro-ondas que é de centímetros. A rugosidade do terreno pode ser lisa ou rugosa. Superfícies lisas causam reflexão especular da energia emitida, aparecendo com tonalidades escuras nas imagens SAR, enquanto as superfícies rugosas espalham a energia emitida de forma mais intensa, aparecendo com tonalidades mais claras nas imagens. O conceito de rugosidade do terreno depende do comprimento de onda e do ângulo de incidência, ou seja, uma superfície pode ser rugosa para comprimentos de onda mais curtos ou ângulos de incidência maiores, ou lisa para comprimentos de onda mais longos ou ângulos de incidência menores. Embora haja poucos estudos sobre estimativa de rugosidade do terreno na literatura, ainda é importante considerar os seus efeitos, por exemplo, em estudos envolvendo estimativa de umidade do solo e biomassa acima do solo (AGB). Em um dos estudos pioneiros conduzidos por Ulaby e Batlivala (1976) usando um escaterômetro portátil, os autores mostraram que a influência da rugosidade do solo é menos pronunciada para ângulos de incidência em torno de $10^{\circ}$. As medições quantitativas e representativas da rugosidade da superfície do solo no campo para validar estimativas derivadas de imagens SAR são bastante complexas e demoradas. Várias medições são necessárias para conseguir um valor representativo de rugosidade do terreno da área de estudo, especialmente se a área for grande ou heterogênea em termos de rugosidade (NURTYAWAN et al., 2020). 
A estrutura da vegetação também afeta fortemente o processo de retroespalhamento. Ela é definida como a proporção de gramíneas, arbustos e árvores presentes em uma área de interesse. Galhos e troncos de arbustos e árvores atuam como dispersores individuais dos sinais de radar emitidos. Portanto, as áreas com maior densidade de arbustos e árvores aparecerão com tonalidades mais claras nas imagens de radar em comparação com outras áreas dominadas pelas espécies gramíneas. Assim, as imagens de radar podem ser usadas para estimar AGB ou para monitorar desmatamentos (vide Seção 6 para mais detalhes). Alguns estudos têm demonstrado que os dados de radar são mais adequados do que os dados ópticos para discriminar fitofisionomias representativas do Cerrado (SANO; FERREIRA; HUETE, 2005) e para mapear florestas secas com forte sazonalidade climática (KOYAMA et al., 2019).

Detalhes adicionais sobre princípios de sensoriamento remoto por radar, tais como geometria de imageamento, resoluções de alcance e de azimute, distorções de relevo, ruído speckle e efeito Doppler podem ser encontrados em diferentes livros didáticos comerciais (por exemplo, HENDERSON; LEWIS, 1998; JENSEN, 2000; SANO; MENESES; ALMEIDA, 2019) ou disponibilizados gratuitamente na internet (por exemplo, CCRS, 2020).

\section{SISTEMAS ORBITAIS}

Após o lançamento do satélite SEASAT, os cientistas tomaram conhecimento do potencial do SAR em diferentes aplicações nos campos da geologia, agricultura, floresta, hidrologia, monitoramento de desastres e oceanografia, entre outras. Recentemente, vários satélites SAR foram lançados e novas missões estão planejadas para futuro próximo. A tendência geral é de que a resolução espacial seja cada vez mais refinada (1-3 metros), embora os dados obtidos com resolução mais grosseira ( $100 \mathrm{~m}$ ) têm sido disponibilizados gratuitamente para permitir estudos globais (OUCHI, 2013). O modo de aquisição de dados em polarização simples está sendo substituída pela capacidade de operação em polarização dupla e até mesmo quádrupla. As principais características das atuais plataformas espaciais de SAR são mostradas no Quadro 1.

O número de países que lançaram satélites com sensores SAR é relativamente pequeno. Canadá, Japão e ESA, organização intergovernamental da Europa com sede em Paris, França, são os que atualmente têm vasta experiência em sensoriamento remoto por radar, desde que lançaram os primeiros satélites na década de 1990, operando com bandas C (Canadá e ESA) e L (Japão). A manutenção das experiências obtidas em faixas específicas de cada país é uma tendência clara. Alemanha e Itália investiram na banda X. Mais recentemente, a Argentina se juntou à comunidade de radar com o lançamento em 2018 de um sensor que opera na banda L e que se encontra a bordo do satélite SAOCOM.

A constelação de dois satélites denominados de Sentinel-1A e Sentinel-1B que operam na banda C e com resolução espacial de 5 metros e periodicidade de 12 dias é único porque se constituem nos únicos dados SAR de alta resolução espacial que estão disponíveis gratuitamente na internet. Esses dois satélites foram lançados em abril de 2014 (Sentinel-1A) e abril de 2016 (Sentinel-2B) no mesmo plano orbital. As imagens podem ser baixadas da plataforma Copernicus Open Access Hub da ESA, mediante cadastro, ou em ambientes de processamento em nuvem como a plataforma Google Earth Engine ${ }^{\mathrm{TM}}$. Vários artigos baseados nos dados Sentinel-1A e Sentinel-1B e envolvendo diferentes aplicações já foram publicados, destacando-se o monitoramento de incêndios florestais (BAN et al., 2020), plantações agrícolas (LA ROSA et al., 2019; MANDAL et al., 2020; SCHLUND; ERASMI, 2020), eventos de inundação (ZHANG et al., 2020) e florestas tropicais (PULELLA et al., 2020). Isso indica que uma política de acesso gratuito é uma das melhores formas de melhorar e aprimorar o uso e aplicação de dados de radar. A combinação dos conjuntos de dados Sentinel1A e Sentinel-1B tem a vantagem de melhorar a resolução temporal para seis dias. 
Quadro 1 - Características de aquisição de imagens dos principais sistemas orbitais SAR de observação da Terra em operação. A polarização e a resolução, que dependem do modo de aquisição da imagem, neste quadro, correspondem às melhores opções de cada satélite. DLR = Agência Aeroespacial da Alemanha; ISA = Agência Espacial da Itália; CSA =

Agência Espacial do Canadá; ESA = Agência Espacial Europeia; S1A = Sentinel-1A; S1B = Sentinel-1B; JAXA = Agência de Exploração Aeroespacial do Japão. CONAE = Agência Espacial da Argentina. A constelação RADARSAT encontra-se em fase de validação e calibração. A resolução temporal dos satélites Sentinel-1A e Sentinel-1B é de 12 dias ou de 6 dias se os dois satélites forem combinados. A JAXA utiliza o plano de observação básica (BOS), designado para seguir uma estratégia específica de aquisição de imagens (JAXA, 2017).

\begin{tabular}{|c|c|c|c|c|c|c|c|}
\hline Satélite & $\begin{array}{c}\text { Agência } \\
\text { (País) }\end{array}$ & $\begin{array}{l}\text { Lança- } \\
\text { mento }\end{array}$ & Banda & Polarização & $\begin{array}{c}\text { Resoluçãa } \\
\text { (m) }\end{array}$ & $\begin{array}{c}\text { Resolução } \\
\text { Temporal } \\
\text { (dias) }\end{array}$ & Citação \\
\hline TerraSAR-X & $\begin{array}{c}\text { DLR } \\
\text { (Alemanha) }\end{array}$ & 2007 & $\mathrm{X}$ & Dupla & 1 & 11 & $\begin{array}{l}\text { Pitz e Miller } \\
(2010)\end{array}$ \\
\hline TanDEM-X & $\begin{array}{c}\text { DLR } \\
\text { (Alemanha) }\end{array}$ & 2010 & $\mathrm{X}$ & Dupla & 1 & 11 & $\begin{array}{l}\text { Krieger et al. } \\
(2007,2013)\end{array}$ \\
\hline COSMO SkyMed & $\begin{array}{c}\text { ISA } \\
\text { (Itália) }\end{array}$ & 2007 & $\mathrm{X}$ & Dupla & 1 & 16 & $\begin{array}{l}\text { Covello et al. } \\
\text { (2010) }\end{array}$ \\
\hline RADARSAT-2 & $\begin{array}{c}\text { CSA } \\
\text { (Canadá) }\end{array}$ & 2007 & $\mathrm{C}$ & Quádrupla & 3 & 24 & $\begin{array}{l}\text { Fox, Luscombe e } \\
\text { Thompson (2004) }\end{array}$ \\
\hline $\begin{array}{l}\text { Constelação } \\
\text { RADARSAT }\end{array}$ & $\begin{array}{c}\text { CSA } \\
\text { (Canadá) }\end{array}$ & 2019 & $\mathrm{C}$ & Quádrupla & 1 & 4 & CSA (2020) \\
\hline $\begin{array}{l}\text { Sentinel-1A e } \\
\text { Sentinel-1B }\end{array}$ & ESA & $\begin{array}{l}2014(\mathrm{~S} 1 \mathrm{~A}) \\
2016(\mathrm{~S} 1 \mathrm{~B})\end{array}$ & $\mathrm{C}$ & Dupla & 5 & $12(6)$ & $\begin{array}{l}\text { Torres et al. } \\
\text { (2012) }\end{array}$ \\
\hline ALOS-2 & $\begin{array}{l}\text { JAXA } \\
\text { (Japão) }\end{array}$ & 2014 & $\mathrm{~L}$ & Quádrupla & 3 & BOS & $\begin{array}{l}\text { Rosenqvist et al. } \\
\text { (2014) }\end{array}$ \\
\hline SAOCOM & $\begin{array}{c}\text { CONAE } \\
\text { (Argentina) }\end{array}$ & 2018 & $\mathrm{~L}$ & Quádrupla & 10 & 16 & ESA (2020) \\
\hline
\end{tabular}

Fonte: Os autores (2020).

A missão TanDEM-X do Centro Aeroespacial da Alemanha (DLR) também é peculiar por causa dos satélites-gêmeos TerraSAR-X e TanDEM-X que orbitam em modo bistático, isto é, os dois sensores adquirem imagens de uma mesma área no terreno simultaneamente e em órbitas próximas, porém, distintas. Os dois satélites foram lançados com o objetivo principal de gerar modelos digitais de elevação (MDEs) de toda a superfície terrestre de forma consistente, com alta resolução espacial e com uma precisão sem precedentes (KRIEGER et al., 2007; RIZZOLI et al., 2017). Recentemente, o conjunto de dados interferométricos TanDEM-X foi usado para produzir um mapa global de floresta/não-floresta (MARTONE et al., 2018). A próxima geração de missões espaciais SAR da DLR irá adotar o conceito de tomografia SAR para adquirir imagens 3D do terreno, após o lançamento da constelação de satélites TanDEM-L (MOREIRA et al., 2015; NANNINI et al., 2019). Este conceito denominado de TanDEM compreende dois satélites de radar polarimétrico com antenas de abertura larga, seguindo o princípio de refletor alimentado por matriz e que permitirá, pela primeira vez, o imageamento de uma faixa contínua de $350 \mathrm{~km}$ no terreno com uma resolução azimutal de $7 \mathrm{~m}$ (HUBER et al., 2018). Diversas aplicações podem se beneficiar da tomografia SAR, incluindo o monitoramento de desmatamento em florestas tropicais, aproveitando-se das diferenças na estrutura tridimensional da floresta. DLR é a única agência espacial que detém esta tecnologia biestática.

\section{R99B SAR}

No início dos anos 2000, a FAB operava dois sensores SAR nas bandas X e L a bordo de aeronaves R99B, com foco principalmente na vigilância da Amazônia brasileira. As imagens foram disponibilizadas nas resoluções espaciais de $3 \mathrm{~m}, 6 \mathrm{~m}$ e $18 \mathrm{~m}$, com polarização quádrupla (HH + HV + HV + VV) para a banda L e polarização simples $(\mathrm{HH})$ para a banda $\mathrm{X}$, com faixas de imageamento de $20 \mathrm{~km}, 40 \mathrm{~km}$ e $120 \mathrm{~km}$.

Vários estudos foram conduzidos com base neste sistema. Imagens simuladas do satélite de radar binacional (Brasil e Alemanha) na banda L, denominado de Multi-Application Purpose SAR (MAPSAR), 
foram obtidas pela aeronave R99B (Mura et al., 2009). Alves et al. (2009) desenvolveram uma abordagem metodológica para identificar pistas de aeronaves ilegais no município de Itaituba, estado do Pará. Outros estudos envolveram discriminação de desmatamento na Amazônia brasileira (GUERRA; MURA; FREITAS, 2010), mapeamento geomorfológico no município de Iranduba, estado do Amazonas (PINTO; LUCHIARI, 2017), mineração de ouro na Província Mineral de Tapajós (CARRINO et al., 2011), lateritas mineralizadas de ferro na Província Mineral de Carajás (SILVA et al., 2013) e discriminação de ambientes úmidos costeiros (SOUZA-FILHO et al., 2011). Infelizmente, o MAPSAR nunca foi lançado e o R99B SAR deixou de ser operacional.

\section{PROCESSAMENTO DE IMAGENS SAR}

Quando os dados brutos SAR são pré-processados, os produtos resultantes estarão em um formato complexo conhecido como single-look complex (SLC), contendo dados de amplitude e fase. A amplitude de cada pixel é proporcional à magnitude do retroespalhamento do sinal emitido e depende da geometria de imageamento, comprimento de onda, polarização e propriedades geométricas e dielétricas dos alvos (OUCHI, 2013). Considerando que existem vários dispersores dentro de uma célula de resolução SAR, cada um dos dispersores retroespalham os sinais emitidos em tempos diferentes, o que é uma característica relacionada à informação de fase na análise de imagens de radar. Fase representa o ponto no comprimento de onda do sinal retroespalhado quando atinge a antena, sendo medido em graus ou radianos. A amplitude é a parte real do valor complexo, enquanto a fase é a parte imaginária. Em termos práticos, a fase de uma imagem pode ser explorada via decomposição polarimétrica, geração de interferogramas ou MDEs. Nesta seção, são apresentados mais detalhes sobre as principais etapas de processamento envolvidas na geocodificação, decomposição de alvos e interferometria de radar de abertura sintética (InSAR).

\subsection{Geocodificação}

Embora os usuários tenham a opção de solicitar imagens SAR já projetadas de alcance inclinado para o alcance no terreno e geocodificadas com base em algum MDE, nesta seção serão apresentadas as etapas mais importantes para converter dados SLC em coeficientes de retroespalhamento ( $\sigma^{\circ}$, unidades em decibéis) e em imagens geocodificadas. A geocodificação é importante para permitir que os dados SAR sejam integrados com outros tipos de dados geoespaciais, por exemplo, outras imagens de satélite ou mapas baseados em vetores. A conversão de dados SLC em dados geocodificados envolve as seguintes etapas:

a) Calibração radiométrica;

b) Filtragem multilook;

c) Filtragem do ruído speckle;

d) Conversão dos valores digitais para $\sigma^{\circ}$; e

e) geocodificação.

Todas as etapas descritas anteriormente podem ser realizadas por meio de programas computacionais de código aberto como o Sentinel Application Platform (SNAP) (ESA, 2019) ou por meio de aplicativos comerciais como o L3 Harris Geospatial ENVI SARScape ${ }^{\mathrm{TM}}$ (SARMAP, 2020). A calibração radiométrica converte os números digitais em coeficientes de retroespalhamento calibrados radiometricamente e geralmente é totalmente automatizada nos aplicativos citados anteriormente ou em qualquer outro aplicativo apropriado, uma vez que todas as informações necessárias estão incluídas no arquivo de metadados dos produtos SAR. Esta etapa é importante especialmente quando o analista está preparando dados para fazer um mosaico de duas ou mais imagens adquiridas em diferentes ângulos de incidência e diferentes níveis de brilho. Em última análise, a calibração radiométrica pode corrigir imagens SAR, de modo que os valores dos pixels efetivamente representem as características dos alvos presentes no terreno (ESA, 2019).

Multilooking é um processo que envolve a conversão de alcance inclinado para alcance no terreno, 
produzindo dados com tamanho nominal do pixel e com menor quantidade de ruído, uma vez que o processo de extração de média aritmética envolvido neste procedimento suaviza as imagens (ENVI, 2020). Por exemplo, um sensor hipotético com uma resolução espacial nominal de $6,25 \mathrm{~m}$ pode ser redimensionado para um tamanho de pixel de 5 metros neste procedimento. Nesse sentido, os dados SAR processados são frequentemente especificados em termos de tamanho de pixel ao invés de resolução espacial nominal. Quando as imagens SLC são filtradas usando um filtro multilook, as imagens de intensidade resultante (amplitude elevada ao quadrado) são chamadas de imagens de potência.

Speckle é um tipo de ruído inerente às imagens SAR, está associado às interferências aleatórias construtivas e destrutivas de dispersores individuais presentes no terreno e causa um aspecto de sal e pimenta nas imagens. Os filtros speckle são aplicados para reduzir o aspecto "borrado" presente nas imagens SAR. Vários filtros estão disponíveis na literatura, por exemplo, os filtros Lee, Frost e Gamma Map. Escolher o melhor filtro speckle não é uma tarefa trivial porque não há nenhuma imagem correspondente sem ruído para ser utilizado como referência. Assim, a qualidade do filtro geralmente é avaliada visualmente em termos da presença ou ausência de desfocamento em áreas homogêneas e preservação de feições do terreno em áreas heterogêneas (ARGENTI; LAPINI; ALAPARONE, 2013).

Valores digitais (VD) são convertidos em $\sigma^{\circ}(\mathrm{dB})$ por meio da seguinte equação (Eq. 1):

$$
\sigma^{\circ}(d B)=-10 * \log \left(V D^{2}\right)+F C
$$

Onde $F C=$ fator de calibração de cada sensor.

Alternativamente, alguns autores utilizam $\gamma^{\circ}$ ao invés de $\sigma^{\circ} ; \gamma^{\circ}$ corresponde ao $\sigma^{\circ}$ corrigido pelos efeitos do ângulo de incidência local ( $\theta^{\prime}$ ) (Eq. 2):

$$
\gamma(d B)=\sigma^{\circ} / \cos \left(\theta^{\prime}\right)
$$

Tipicamente, os valores de $\sigma^{\circ}$ variam de $-32 \mathrm{~dB}$ a $+16 \mathrm{~dB}$. Os valores provenientes de polarização paralela geralmente são mais altos em relação aos provenientes da polarização cruzada. Os valores mais baixos são normalmente encontrados em alvos que causam reflexão especular da energia emitida, por exemplo, corpos d'água límpidos sem vento. Por outro lado, os valores de $\sigma^{\circ}$ mais altos são encontrados em alvos que causam reflexão de canto, por exemplo, áreas urbanas com edifícios distribuídos perpendicularmente à direção de alcance do sensor.

A geocodificação requer o uso de um MDE para a correção do terreno e para inserir um sistema de coordenadas no produto final. Embora os aplicativos mencionados anteriormente tenham seus próprios modelos, os usuários podem escolher outros modelos de elevação. Os MDEs mais comuns disponíveis gratuitamente na internet incluem o Shuttle Radar Topography Mission (SRTM-3 Versão 4, resolução de 30 m) e o ASTER Global DEM (GDEM). A NASA lançou um novo produto denominado de NASA DEM (resolução de 30 metros) sem muitas das limitações e restrições presentes no produto SRTM DEM e com maior precisão e cobertura de área. A JAXA também lançou uma nova versão de MDE, denominado de ALOS World 3D-30m (AW3D30), produzido com base no sensor óptico pancromático PRISM a bordo do satélite ALOS-1 que operou de 2006 a 2011 (TAKAKU; TADONO; TSUTSUI, 2014). O DLR produziu um MDE global com precisão sem precedentes (resolução horizontal de $12 \mathrm{~m}$ e precisão de altura relativa de $2 \mathrm{~m}$ ) usando os satélites TerraSAR-X e TanDEM-X que operam em planos orbitais distintos e próximos (ZINK et al., 2014). Este produto, entretanto, não se encontra disponível para download na internet de forma gratuita.

A Figura 2 ilustra um exemplo de uma imagem de radar com polarização HH de uma região de ecótono localizada entre os biomas Amazônia e Cerrado, mais especificamente, no município de Sinop, estado do Mato Grosso e arredores, adquirida pelo satélite ALOS-2 em fevereiro de 2016, no âmbito da Iniciativa Kyoto \& Carbono da JAXA (ROSENQVIST et al., 2010). A imagem foi adquirida no modo Stripmap, resolução espacial de 6,25 m e formato CEOS. A Figura 2A mostra a imagem obtida em alcance inclinado. Percebe-se a presença de distorções geométricas que reduzem a utilidade desta imagem, muitas vezes impedindo a extração 
de informações. A Figura 2B mostra a mesma imagem processada por calibração radiométrica, filtragem multilook, filtragem do ruído speckle por meio do filtro Gamma Map e geocodificação usando os dados do SRTM de $30 \mathrm{~m}$. A imagem foi projetada no sistema de projeção Universal Transversa de Mercator (UTM), fuso UTM = 21S e datum = WGS84. Observa-se que as imagens SLC são orientadas norte-sul, enquanto as imagens geocodificadas são orientadas na órbita ascendente ou descendente do satélite. Mais detalhes sobre o pré-processamento desta imagem podem ser encontrados em Silva et al. (2020).

Figura 2 - Ilustração de uma imagem de satélite ALOS-2/PALSAR-2 adquirida em 12 de fevereiro de 2016 no formato single look complex (A) e processada em termos de calibração radiométrica, filtragens multilook e speckle e geocodificação por meio do modelo digital de elevação obtido pelo SRTM com resolução espacial de 30 m (B).

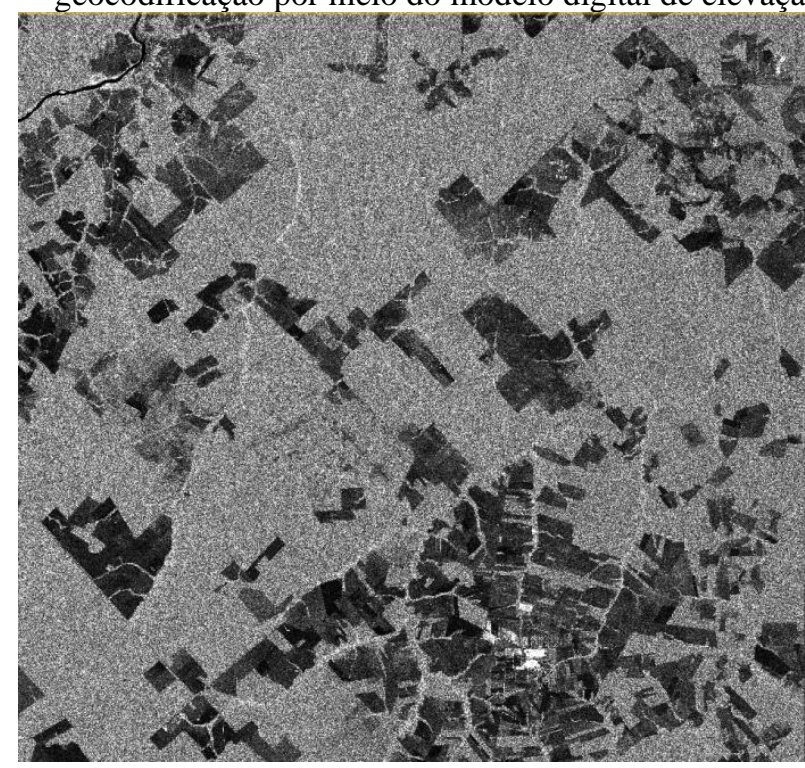

(A)

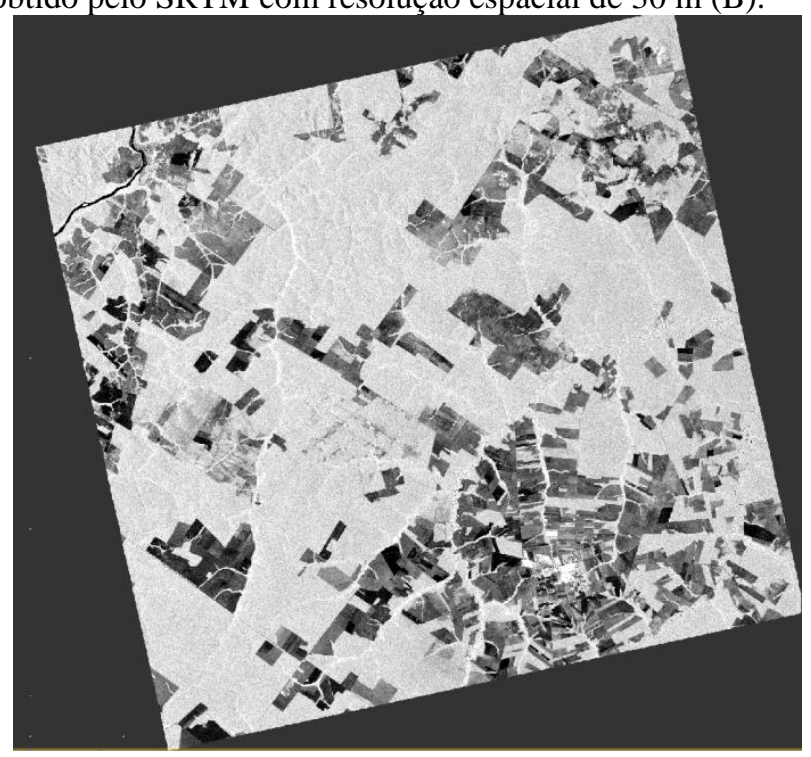

(B)

Fonte: Os autores (2020).

\subsection{Polarimetria e decomposição de alvos}

Os dados SAR polarimétricos mostram uma grande quantidade de informações de alvos terrestres quando comparados com os dados convencionais de amplitude e dados SAR de polarização simples ou dupla (VAN DER SANDEN, 1997; OUCHI, 2013). Isso ocorre porque a polarimetria SAR tem a capacidade de descrever e armazenar dados sobre as mudanças no estado de polarização do vetor do campo elétrico $\vec{E}$ alterado pela estrutura e pela $\varepsilon$ dos objetos presentes na superfície da Terra (LEE; POTTIER, 2009). A equação de uma elipse é a forma mais comum de representar $\vec{E}$ (HELLMANN; 2001; LEE; POTTIER, 2009; RICHARDS, 2009). A matriz de dispersão $S$ (Eq. 3), ou matriz de Jones, é usada para descrever a transformação linear das mudanças que ocorrem entre o vetor elétrico transmitido pela antena SAR e o vetor elétrico espalhado pelos alvos. Esta matriz permite descrever o alvo e os processos físicos atuantes (HELLMANN, 2001).

$$
[S]=\left[\begin{array}{ll}
S_{h h} & S_{h v} \\
S_{v h} & S_{v v}
\end{array}\right] \rightarrow \vec{k}=\frac{1}{2} \operatorname{Traço}([S] \Psi)=\left[k_{0}, k_{1}, k_{2}, k_{3}\right]^{T}
$$

Onde $[S]$ é a matriz de espalhamento $\mathrm{S} ; S_{i j}$ é o elemento complexo de [S]; $k_{i}$ é o elemento complexo do vetor de Jones $(\vec{k})$; Traço [S] é a soma dos elementos na diagonal de [S]; e $\Psi$ é matriz de base $2 \times 2$.

A base de Pauli é utilizada para estimar $\Psi$ (Eq. 4) (HELLMANN, 2001; LEE; POTTIER, 2009):

$$
\Psi_{P}=\left\{\sqrt{2}\left[\begin{array}{ll}
1 & 0 \\
0 & 1
\end{array}\right], \sqrt{2}\left[\begin{array}{cc}
0 & 1 \\
0 & -1
\end{array}\right], \sqrt{2}\left[\begin{array}{ll}
0 & 1 \\
1 & 0
\end{array}\right]\right\}
$$

Alternativamente, a base de Borgeaud pode ser utilizada para estimar $\Psi$ (Eq. 5): 


$$
\Psi_{B}=\left\{2\left[\begin{array}{ll}
1 & 0 \\
0 & 0
\end{array}\right], 2 \sqrt{2}\left[\begin{array}{ll}
0 & 1 \\
0 & 0
\end{array}\right], 2\left[\begin{array}{ll}
0 & 0 \\
1 & 0
\end{array}\right]\right\}
$$

Lee e Pottier (2009) introduziram o conceito de alvos distribuídos, ou seja, na imagem SAR, nem todos os alvos são estacionários ou fixos e podem variar ao longo do tempo. Os alvos naturais, por exemplo, mudam devido à dinâmica do vento, temperatura e pressão. Portanto, a onda retroespalhada deve ser considerada como parcialmente polarizada, o que significa que a onda não é mais coerente, monocromática e totalmente polarizada na forma descrita pela elipse de polarização. Assim, a matriz de espalhamento $S$ apresenta limitações para representar os alvos incoerentes, requerendo matrizes de covariância e coerência, também chamadas de matrizes de potência, para encontrar áreas com predomínio de alvos distribuídos. A matriz de covariância polarimétrica é formada pelo cruzamento entre o vetor de Borgeaud e o seu complexo conjugado transposto (Eq. 6) (HELLMANN, 2001):

$$
[C]_{4 x 4}=\left\langle\vec{k}_{B} \vec{k}_{B}^{*} T=\left[\begin{array}{cccc}
\left\langle\left|S_{h h}\right|^{2}\right\rangle & \left\langle S_{h h} S_{h v}^{*}\right\rangle & \left\langle S_{h h} S_{v h}^{*}\right\rangle & \left\langle S_{h h} S_{v v}^{*}\right\rangle \\
\left\langle S_{h v} S_{h h}^{*}\right\rangle & \left\langle\left|S_{h v}\right|^{2}\right\rangle & \left\langle S_{h v} S_{v h}^{*}\right\rangle & \left\langle S_{h v} S_{v v}^{*}\right\rangle \\
\left\langle S_{v h} S_{h h}^{*}\right\rangle & \left\langle S_{v h} S_{h v}^{*}\right\rangle & \left\langle\left|S_{v h}\right|^{2}\right\rangle & \left\langle S_{v h} S_{v v}^{*}\right\rangle \\
\left\langle S_{v v} S_{h h}^{*}\right\rangle & \left\langle S_{h h} S_{h v}^{*}\right\rangle & \left\langle S_{v v} S_{v h}^{*}\right\rangle & \left\langle\left|S_{v v}\right|^{2}\right\rangle
\end{array}\right]\right.
$$

Onde $[C]_{4 \times 4}$ é a matriz $4 \times 4$ de covariância; os elementos diagonais representam a intensidade do sinal na respectiva polarização; $\langle\ldots\rangle$ é a média espacial; $k_{B}$ é o vetor de Borgeaud; e $\vec{k}_{B}^{*} T$ é o complexo conjugado do vetor de Borgeaud.

Outro conceito de lidar com a natureza não determinística ou incoerente da onda retroespalhada consiste em estimar uma matriz de coerência polarimétrica (HELLMANN, 2001). Esta matriz é formada pelo produto do cruzamento entre o vetor de Pauli e o seu complexo conjugado (Eq. 7):

$$
[T]_{4 \times 4}=\left\langle\vec{k}_{P} \vec{k}_{P}^{*} T\right\rangle
$$

Ao assumir o teorema da reciprocidade, pode-se descrevê-lo como $S_{h v}=S_{v h}$. Nesse caso, o vetor de Pauli pode ser reduzido a três dimensões sem perda de informações (Eq. 8) (HELLMANN, 2001):

$$
[T]_{3 \times 3}=\left\langle\vec{k}_{P_{3}} \vec{k}_{P_{3}}^{*}\right\rangle=\frac{1}{2}\left[\begin{array}{ccc}
\left\langle|A|^{2}\right\rangle & A B^{*} & A C^{*} \\
\left\langle A^{*} B\right\rangle & \left\langle|B|^{2}\right\rangle & B C^{*} \\
\left\langle A^{*} C\right\rangle & \left\langle B^{*} C\right\rangle & \left\langle|C|^{2}\right\rangle
\end{array}\right]
$$

Onde $[T]_{3 \times 3}$ é a matriz de coerência $3 \times 3 ; k_{P}$ é o vetor de Pauli; $\vec{k}_{P}^{*} T$ é o complexo conjugado transposto do vetor de Pauli; $A=S_{h h}+S_{v v} ; B=S_{h h}-S_{v v}$; e $C=2 S_{h v}$.

As técnicas de decomposição visam representar o mecanismo de espalhamento médio como uma soma de elementos independentes, associando um mecanismo de espalhamento físico a cada um desses elementos. Teoremas de decomposição de alvos podem ser classificados em dois grupos principais, decomposição coerente e decomposição incoerente. Decomposições coerentes assumem a ocorrência de dispersores determinísticos e a onda retroespalhada é polarizada. Este tipo de decomposição usa a matriz de espalhamento $S$ para representar o estado de polarização da onda retroespalhada. Por outro lado, as decomposições incoerentes consideram a priori que o espalhamento não é do tipo determinístico e a onda retroespalhada é parcialmente polarizada. Nessa situação, matrizes de covariância e coerência são utilizadas para caracterizar a onda retroespalhada (LEE; POTTIER, 2009; RICHARDS, 2009).

Em aplicações de sensoriamento remoto, a premissa de ocorrência de alvos puramente determinísticos nem sempre é válida (RICHARDS, 2009). Assim, as matrizes de reflexão de potência são frequentemente utilizadas. Com base nessas matrizes, este último autor descreveu apenas os métodos de decomposição de 
alvos incoerentes, com ênfase nos seguintes teoremas de decomposição de alvos: Yamaguchi, com quatro componentes; e entropia (H), anisotropia (A) e ângulo $\alpha$, associados com decomposição de Cloude-Pottier. O processamento de imagens envolvendo esses teoremas pode ser realizado, por exemplo, no aplicativo SNAP. As imagens devem estar no formato SLC e no modo polarimétrico (CAMARGO et al., 2019). No caso da decomposição de Cloude-Pottier, é possível gerar decomposições de imagens SLC com polarização dupla usando o aplicativo SNAP. O processamento da imagem é aplicado diretamente aos dados do SLC sem qualquer pré-processamento do tipo calibração ou filtragem, tornando o uso de filtros polarimétricos opcional para remover o ruído speckle. O uso cuidadoso de filtros polarimétricos nas matrizes é recomendado para evitar reamostragem desnecessária e perda de informações úteis.

Yamaguchi et al. (2005) desenvolveram um modelo de decomposição de alvos baseado em modelagem de matriz de covariância. Esse modelo consiste de quatro componentes de espalhamento: reflexão de canto, espalhamento superficial, espalhamento volumétrico e hélice. Os três primeiros componentes são baseados em seus correspondentes já apresentados no modelo de Freeman e Durden (1998). O quarto componente foi introduzido por Yamaguchi et al. (2005) e estima o espalhamento helicoidal, que é um alvo com forma e/ou estrutura artificial, definido como um refletor assimétrico capaz de gerar polarizações circulares à esquerda ou à direita, sendo mais adequado para descrever alvos artificiais.

Yamaguchi et al. (2005) também modificaram a modelagem de espalhamento volumétrico para melhor representar o espalhamento em ambientes florestais. O modelo teórico convencional de espalhamento volumétrico consiste em uma nuvem de dipolos de orientação aleatória com modelagem baseada em uma função de densidade de probabilidade uniforme em diferentes ângulos de orientação. Porém, em ambientes predominantemente ocupados por estruturas verticais como é o caso de formações florestais, as dispersões de troncos e galhos apresentam ângulos de distribuição preferenciais. Esses autores ajustaram a função de densidade de probabilidade de Freeman-Durden considerando alguns aspectos da orientação dominante dos troncos e galhos das árvores. As Figuras 3C e 3D mostram as composições coloridas geradas pelos componentes derivados do teorema de Yamaguchi.

A decomposição de Cloude-Pottier pode ser usada para analisar autovalores e autovetores da matriz de coerência por meio da decomposição dessa matriz em elementos que representam espalhamento parcial. Essa decomposição incluirá todos os tipos de mecanismos de espalhamento, com foco na análise de autovalores e autovetores que tem a vantagem de serem independentes da base e sempre ser diagonalizável (CLOUDE; POTTIER, 1997; LEE; POTTIER, 2009). Na proposta de Cloude e Pottier, a matriz de coerência é decomposta pela soma de três outras matrizes de coerência parciais e cada matriz individual é ponderada por seu respectivo autovalor (Eq. 9):

$$
\left[T_{3}\right]=\sum_{i=1}^{i=3} T_{3_{i}}=\sum_{i=1}^{i=3} \lambda_{i}\left(\vec{e}_{i} \cdot \vec{e}_{i}^{* T}\right)=\lambda_{1}\left(\vec{e}_{1} \cdot \vec{e}_{1}^{* T}\right)+\lambda_{2}\left(\vec{e}_{2} \cdot \vec{e}_{2}^{* T}\right)+\lambda_{3}\left(\vec{e}_{3} \cdot \vec{e}_{3}^{* T}\right)
$$

Onde $\left[T_{3}\right]$ corresponde à matriz de coerência $3 \times 3 ; \lambda_{i}$ é o autovalor; e $e_{i}$ corresponde ao autovetor.

Cada uma das matrizes individuais representa uma contribuição de um tipo específico de espalhamento determinístico. O peso de cada contribuição é dado pelo autovalor, enquanto o tipo de mecanismo de espalhamento é dado pelo autovetor. Os principais indicadores derivados do teorema da decomposição do alvo são entropia (H), anisotropia (A) e medição do ângulo $\alpha$ (CLOUDE; POTTIER, 1997; LEE; POTTIER, 2009). A Figura 3B mostra uma composição colorida gerada com os componentes derivados do teorema de decomposição de Cloude-Pottier. A entropia polarimétrica $(\mathrm{H})$ indica o grau de aleatoriedade na distribuição dos mecanismos de espalhamento (Eq. 10):

$$
H=-\sum_{i=1}^{3} P_{i} \log _{3} P_{i}, \quad P_{i}=\frac{\lambda_{i}}{\sum_{k=1}^{3} \lambda_{k}}
$$

Figura 3 - Composições coloridas RGB apresentando: A) imagens polarizadas em amplitude (HH/HV/VV); B) Teorema da decomposição de Cloude-Pottier (H/A/ $\alpha)$; C) Teorema de Yamaguchi sem o componente de hélice 
(duplo/volume/superfície); e D) Teorema de Yamaguchi com a componente de hélice (dupla/hélice/superfície). As imagens correspondem a uma cena ALOS-2/PALSAR-2 sobre parte do município de Planaltina, estado de Goiás, adquirida em 16 de maio de 2016, em órbita ascendente, polarização quádrupla e com resolução espacial de 6,25 m.

Datum horizontal: WGS84; sistema de projeção: UTM 23S.
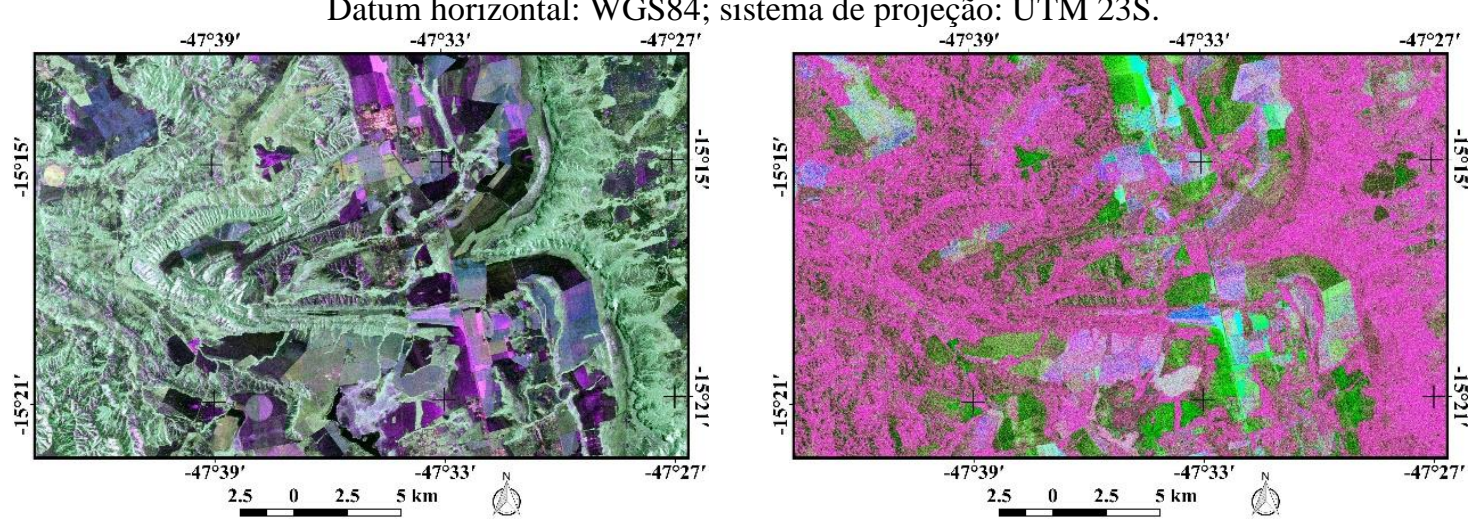

(A)

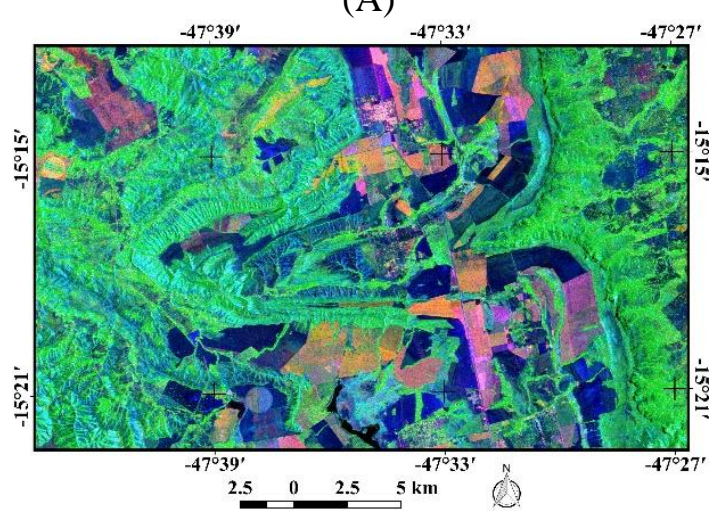

(B)

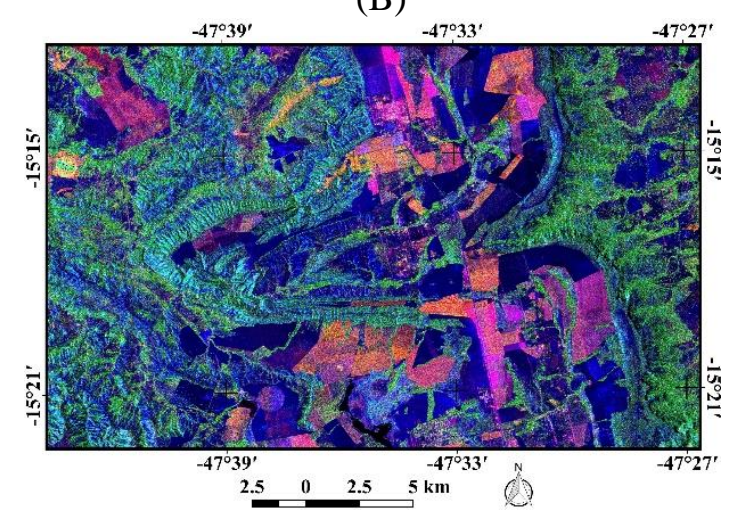

(C)

(D)

Fonte: Os autores (2020).

Os valores de $P i$ são interpretados como a intensidade relativa do processo de espalhamento $i$. Os valores de $\mathrm{H}$ variam entre 0 e $1 ; \mathrm{H}=0$ indica que a matriz de coerência tem apenas um autovalor $\left(\lambda_{i}\right)$ diferente de 0 , representando apenas um processo de espalhamento determinístico. Por outro lado, $\mathrm{H}=1$ indica que os autovalores da matriz de coerência são todos iguais, com um processo de espalhamento do tipo ruído aleatório que despolariza totalmente a onda incidente (CLOUDE; POTTIER, 1997; LEE; POTTIER, 2009). A anisotropia polarimétrica é um indicador da importância relativa dos mecanismos de espalhamento secundário, que é mais representativa quando ocorre entropia média, dada por (Eq. 11):

$$
A=\frac{\lambda_{2-} \lambda_{3}}{\lambda_{2+} \lambda_{3}}
$$

De acordo com Cloude e Pottier (1997) e Lee e Pottier (2009), nos casos de alta entropia, a anisotropia não fornece nenhuma informação adicional, visto que os autovalores são aproximadamente iguais. Em casos de baixa entropia, os valores são próximos de zero. A entropia média indica que mais de um mecanismo de espalhamento está contribuindo para o retroespalhamento, mas não estima a quantidade de mecanismos. Anisotropia alta indica que apenas o segundo mecanismo de espalhamento é importante, enquanto a anisotropia baixa indica que também há contribuição do terceiro mecanismo de espalhamento. A partir da matriz de coerência, cada autovetor também pode ser parametrizado em termos de cinco ângulos, $\alpha, \beta, \varphi, \delta$ e $\gamma$, com ênfase no ângulo $\alpha$, com variação entre $0^{\circ}$ e $90^{\circ}$, uma vez que identifica o tipo de mecanismo de espalhamento do alvo. Para $\alpha$ próximo de $0^{\circ}$, há um predomínio do espalhamento isotrópico de salto ímpar; para $\alpha$ próximo de $45^{\circ}$, predomina o espalhamento dipolo; e para $\alpha$ próximo de $90^{\circ}$, predomina a reflexão de canto (CLOUDE; POTTIER, 1997; LEE; POTTIER, 2009). 


\subsection{InSAR}

InSAR consiste no uso de duas ou mais imagens SLC da mesma área geográfica com diferentes geometrias de aquisição para extrair diferenças de fase em cada pixel (HANSSEN, 2002; RICHARDS, 2009). A diferença de fase obtida entre imagens complexas está relacionada à distância entre as duas trajetórias durante cada aquisição de imagem, ao comprimento da linha de base, isto é, a distância entre as antenas, à altura do objeto no solo e ao comprimento de onda do sistema SAR. Assim, obtém-se o ângulo da fase interferométrica e, posteriormente, as elevações do terreno.

Antes do processamento InSAR, deve-se obter um registro preciso entre as imagens SLC. Também é necessário filtrar as imagens na direção de alcance para remover informações espectrais incomuns nas imagens relacionadas às diferenças na linha de visada de cada antena. Este procedimento permite a remoção de ruído em imagens de fase interferométrica. O interferograma é um dos principais produtos do processamento InSAR. Devido às características cíclicas da fase interferométrica, os interferogramas apresentam faixas de altura do objeto a cada ciclo de $2 \pi$ (HANSSEN, 2002; RICHARDS, 2009).

Depois de gerar o interferograma, as variações de fase relacionadas às mudanças nos ângulos de incidência ao longo do alcance da antena do sistema SAR devem ser removidas. Além disso, deve-se gerar ainda a imagem de coerência interferométrica para avaliar a qualidade do interferograma em relação a possíveis erros devido a decorrelações temporais, ruído e decorrelações espaciais causadas pela linha de base da antena. Finalmente, para gerar o MDE, o gradiente das fases precisa ser integrado ao longo da direção de alcance, adicionando ou subtraindo $2 \pi$ para cada descontinuidade. Para a obtenção de dados de elevação do terreno, pontos de controle com coordenadas planimétricas e altimétricas conhecidas são usados para calibrar o interferograma (HANSSEN, 2002; RICHARDS, 2009).

\section{EXEMPLOS DE APLICAÇÃO}

O Quadro 2 resume algumas das aplicações de dados SAR, variando de geologia à criosfera. Não há pretensão de apresentar uma lista completa de inscrições; ao invés disso, estão sendo enfatizados alguns comentários sobre as aplicações potenciais de sensoriamento remoto por radar para aqueles que não estão muito familiarizados com esta tecnologia.

Quadro 2 - Uso potencial de dados SAR em diferentes aplicações.

\begin{tabular}{|l|l|}
\hline \multicolumn{1}{|c|}{ Aplicação } & \multicolumn{1}{c|}{ Especificação } \\
\hline Geologia & $\begin{array}{l}\text { Geologia estrutural, prospecção mineral, geração de MDE, } \\
\text { monitoramento de deslizamentos } \\
\text { Estimativa de biomassa, monitoramento de desmatamento } \\
\text { Floresta }\end{array}$ \\
Agricultura & $\begin{array}{l}\text { Estimativa de umidade de solos, inundação } \\
\text { Hidrologia } \\
\text { Oceanografia } \\
\text { Criosfera }\end{array}$ \\
\hline
\end{tabular}

Fonte: Os autores (2020).

\subsection{Detecção de embarcações e derrames de óleo}

As detecções de embarcações e derramamentos de óleo estão entre as principais aplicações dos dados SAR, especialmente envolvendo a banda C. Uma quantidade substancial de artigos tem sido publicada sobre essas duas aplicações (LI; HE; WANG, 2009; MIGLIACCIO et al., 2012; DECHESNE et al., 2019). A pesquisa sobre detecção de embarcações está principalmente relacionada à verificação de requisitos marítimos internacionais, identificação de embarcações em alguma operação ilegal e vigilância da pirataria (OUCHI, 2013). Por exemplo, grande parte da poluição de óleo no mar relacionada com embarcações está associada a operações de rotina como lavagem de tanques ao invés de acidentes (ALPERS; HOLT; ZENG, 2017). A principal desvantagem da identificação de embarcações por SAR é a resolução espacial relativamente baixa 
dos sistemas orbitais atualmente disponíveis para este tipo de aplicação.

A poluição da superfície do mar associada com exploração de petróleo também tem provocado grandes preocupações ambientais devido a acidentes como o do Golfo do México em 2010 (LAVROVA; KOSTIANOY, 2011; LEIFER et al., 2012). Portanto, o monitoramento continuado das atividades de exploração de petróleo pelas plataformas marítimas é essencial para evitar grandes acidentes ambientais. A tensão superficial do óleo é maior do que a da água, de modo que manchas de óleo observadas nas superfícies do oceano reduzem drasticamente as ondas de Bragg em escala centimétrica, responsáveis pelo retroespalhamento do radar, causando reflexão especular sobre os sinais de micro-ondas incidentes. Consequentemente, os derramamentos de óleo aparecem como manchas escuras no oceano. Comprimentos de onda menores, como as bandas $\mathrm{X}$ e C, são mais adequados para esse propósito, pois o efeito de amortecimento das ondas de Bragg é maior para comprimentos de onda menores. A polarização VV é preferível à polarização HH devido ao seu maior processo de retroespalhamento na superfície do mar (VALENZUELA, 1978). No entanto, o desenvolvimento de algoritmos para identificar vazamentos de óleo de forma inequívoca em imagens de radar não é uma tarefa trivial. De acordo com Alpers, Holt e Zeng (2017), existem 13 fontes potenciais de fenômenos que podem estar relacionados com manchas escuras no oceano, notadamente, o plâncton e os ventos fracos a moderados. Se a velocidade do vento for inferior a 3-4 $\mathrm{m} \mathrm{s}^{-1}$, a superfície do mar também pode aparecer escura, dificultando a identificação de vazamento de óleo (IVANOV, 1998). Se manchas escuras estiverem ligadas a plataformas de petróleo offshore, como mostrado na Figura 4, as chances de manchas estarem relacionadas a plânctons ou ventos ficam reduzidas.

Figura 4 - Imagem de radar do satélite Sentinel-1B obtida em 4 de setembro de 2019 na polarização VV, mostrando feições suspeitas de derramamento de óleo associada à plataforma de exploração de petróleo na bacia de Campos, litoral norte do estado do Rio de Janeiro.

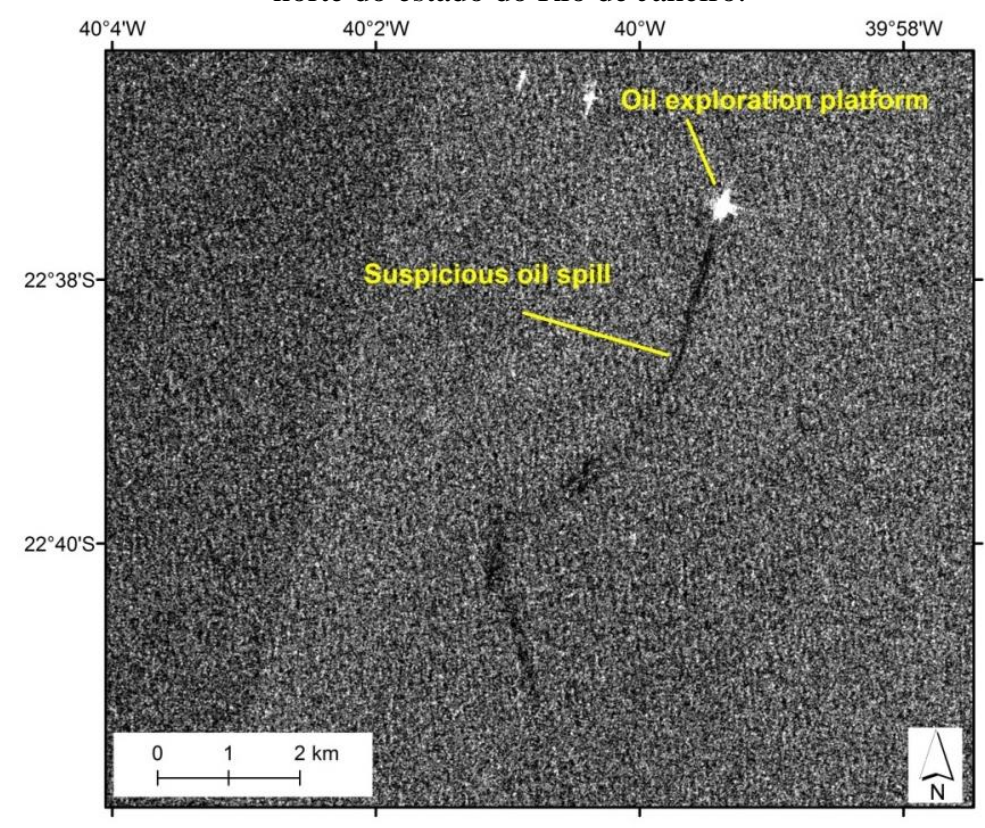

Fonte: Os autores (2020).

\subsection{Monitoramento florestal}

Os produtos SAR também podem ser utilizados para extrair informações florestais, principalmente em países tropicais, incluindo estimativa de estoques de carbono acima do solo e monitoramento de desmatamento por corte raso, corte seletivo de madeira e regeneração secundária. Para estimativa de estoque de carbono, a regra geral é que os sinais retroespalhados aumentam com o aumento dos níveis de biomassa. Comprimentos de onda mais longos penetram mais profundamente nas florestas, de modo que a banda $\mathrm{L}$ geralmente fornece estimativas de biomassa mais precisas, uma vez que ramos e troncos são os principais constituintes da AGB. Os processos de espalhamento dominantes nas bandas X e C ocorrem no topo das camadas do dossel. Também é conhecido que a polarização cruzada se correlaciona melhor do que a polarização paralela com a biomassa 
das árvores. A polarização HV é a mais sensível e a VV, a menos sensível (KASISCHKE; MELACK; DOBSON, 1997).

Devido à relevância da Amazônia brasileira em termos de conservação da biodiversidade e mitigação das mudanças climáticas, vários estudos que avaliaram o potencial da tecnologia SAR para detectar e monitorar o desmatamento tem sido publicados (ALMEIDA-FILHO et al., 2005; PEREIRA et al. , 2016; HAGENSIEKER; WASKE, 2018). Áreas desmatadas consolidadas aparecem como manchas escuras nas imagens SAR devido à diminuição de AGB, conteúdo de água da planta e dispersão de volume de detritos de madeira no solo (Figura 5). No entanto, dependendo do intervalo de tempo entre o desmatamento e a data de passagem do satélite, as áreas desmatadas podem aparecer brilhantes em imagens de radar, principalmente na polarização HH. Isso ocorre porque os detritos de madeira (troncos e galhos) que ficam no solo aumentam os processos de retroespalhamento do radar para valores superiores aos encontrados nas florestas primárias circundantes (ALMEIDA-FILHO et al., 2005). Pesquisa conduzida por Watanabe et al. (2018) mostrou que $\sigma_{H H}^{\circ}$ aumentou 1,1-2,5 dB em áreas com desmatamento em estágio inicial, enquanto $\sigma_{H V}^{\circ}$ diminuiu $0,7-1,0 \mathrm{~dB}$. Portanto, qualquer sistema de monitoramento em tempo quase real de desmatamento com base no conjunto de dados SAR, por exemplo, o JICA-JAXA Forest Early Warning System in the Tropics (JJ-FAST), não deve contar apenas com a polarização HV. Outro estudo de Reiche et al. (2015) indicou que a razão HV/HH forneceu resultados mais precisos quando as áreas desmatadas foram comparadas com o índice de vegetação por diferença normalizada (NDVI) derivada do satélite Landsat.

Figura 5 - Imagem do satélite ALOS-2 PALSAR-2 da região de Sinop, estado de Mato Grosso, adquirida em 12 de fevereiro de 2016 na polarização HH e tamanho do pixel de $6,25 \mathrm{~m}$. Os tons cinza claro correspondem principalmente a florestas tropicais primárias de terras altas, o cinza escuro corresponde ao desmatamento consolidado e o tom claro no interior do círculo vermelho na imagem do ALOS corresponde à sede do município de Cláudia.

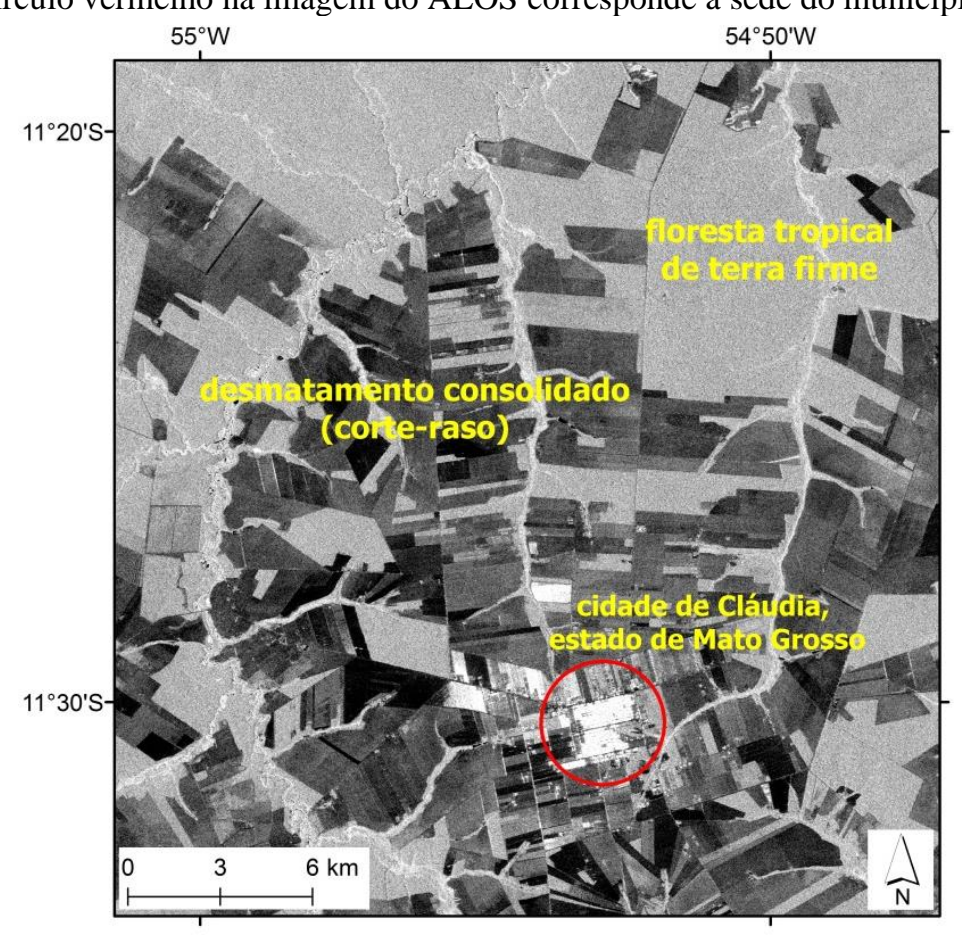

Fonte: Os autores (2020).

A extração seletiva de madeiras tem aumentado substancialmente nas regiões tropicais. Perturbações florestais por atividades madeireiras variam de acordo com as características da floresta, intensidade da exploração e técnicas de colheita (DYKSTRA, 2001; TRITSCH et al., 2016) e podem impactar severamente os ecossistemas florestais e aumentar a susceptibilidade da floresta ao fogo (MONTEIRO et al., 2004; MATRICARDI et al., 2013; THOMPSON et al., 2013). Portanto, é um tipo de atividade antrópica importante de ser monitorado em florestas tropicais, comumente caracterizado por alta cobertura de nuvens. Os produtos SAR podem ser úteis para detectar as florestas afetadas pelas atividades madeireiras em regiões tropicais. Jackson e Adam (2020) relataram um total de 20 artigos científicos usando sensores aerotransportados, 
TerraSAR-X, ALOS-PALSAR, ENVISAT ASAR, TanDEM-X, Radarsat-2 e COSMOSkyMed para investigar a extração seletiva de madeiras em todo o mundo. A maioria desses estudos baseou-se em produtos SAR e alcançou alta precisão geral para mapear florestas afetadas pela extração seletiva. No entanto, eles se concentraram em locais de estudo específicos. Uma avaliação mais abrangente dos distúrbios florestais por extração seletiva ainda precisa ser conduzida e aplicada nos trópicos. Mudanças sutis no dossel e na capacidade de penetração além do dossel da floresta parecem ser a principal restrição e o principal desafio técnico no uso de produtos SAR para detectar impactos florestais decorrentes de atividades madeireiras.

\subsection{Monitoramento de arroz irrigado}

O arroz é um importante alimento básico em diferentes países da Ásia, tais como Bangladesh, China, Indonésia, Japão, Vietnã e outros. Os campos de cultivo semiaquático de arroz cobrem mais de 3 milhões de hectares na China (WU et al., 2020). Esses campos também possuem valor cultural, visto que muitos foram reconhecidos como Patrimônio Agrícola de Importância Mundial (LU; LI, 2006). No Brasil, a produção de arroz irrigado é encontrada principalmente nos estados do Rio Grande do Sul e Santa Catarina (STEINMETZ; BRAGA, 2001). A desvantagem está no aquecimento global, já que os campos de arroz são uma importante fonte de metano, o segundo gás de efeito estufa mais influente nas mudanças climáticas, depois do $\mathrm{CO}_{2}$. Os dados SAR têm sido intensamente usados para detecção, mapeamento e estimativa de produção de campos de arroz (KUROSU; FUJITA; CHIBA, 1995; LE TOAN et al., 1997; SINGHA et al., 2019). Os dados SAR na banda $\mathrm{C}$ e polarização HV se correlacionaram significativamente com o ciclo de crescimento das plantações de arroz (WU et al., 2011; YANG et al., 2012). São necessárias pelo menos três imagens durante a estação de cultivo do arroz para estimar adequadamente os rendimentos (WU et al., 2020). Nesse caso, os dados SAR possuem um bom potencial devido à sua independência das condições de nuvem, já que o ciclo do arroz geralmente se sobrepõe à estação chuvosa. Ishitsuka (2018), por outro lado, afirmou que são necessárias apenas duas imagens, uma detectando áreas de água e outra sem detecção de água. Os campos que exibem este padrão temporal podem ser associados a arrozais plantados com arroz. Durante o pico do ciclo de crescimento, os campos de arroz aparecem brilhantes nas imagens SAR por causa dos efeitos de reflexão de canto devido à presença de hastes de arroz e alagamento (Figura 6). Assim, tais características podem ser adicionadas na abordagem desenvolvida por Ishitsuka (2018) para aumentar a precisão da identificação de campos de arroz.

\subsection{Estimativa de umidade de solos}

A estimativa da umidade do solo é um tema prioritário em hidrologia, uma vez que é um dos parâmetros cruciais no controle da interação entre atmosfera e superfície do solo. No entanto, sua estimativa usando tecnologia SAR tem sido, regra geral, decepcionante. Vários estudos foram conduzidos na década de 1990 usando modelos teóricos, empíricos e semi-empíricos para estimar umidade do solo em diferentes condições climáticas, desde o semi-árido até o temperado úmido (ALTESE; BOLOGNANI; MANCINI, 1996 ; ULABY; DUBOIS; VAN ZYL, 1996; SANO et al., 1998b). Os modelos teóricos mais reconhecidos são o modelo de equação integral e o modelo de pequena perturbação, mas raramente são aplicados devido ao seu baixo desempenho sem o conhecimento prévio de outros parâmetros como rugosidade do solo (OUCHI, 2013). Modelos empíricos e semi-empíricos são geralmente específicos do local, são dependentes dos parâmetros de imageamento de radar e exigem uma grande quantidade de dados in situ. Minimizar os efeitos da rugosidade do solo e da cobertura vegetal não é uma tarefa fácil. Na realidade, os resultados não têm sido satisfatórios quando os sistemas imageadores não possuem capacidade de operação com polarização quádrupla. Além disso, dados precisos de verdades de campo para validação são difíceis de serem obtidos devido à alta variabilidade espacial e temporal desse parâmetro. No entanto, algumas iniciativas recentes têm insistido nesse assunto, como a liderada por Nicolas Baghdadi e colaboradores da Universidade de Montpellier, França (BAGHDADI; HOLAH; ZRIBI, 2006; BAGHDADI et al., 2016; EL HAJJ; BAGHDADI; ZRIBI , 2019). Basicamente, esses autores estão colocando esforços em dados SAR obtidos com diferentes ângulos de visada e frequência para obter melhores estimativas de umidade do solo. 
Figura 6 - Cena do satélite RADARSAT-1 adquirida em 4 de fevereiro de 1999 com resolução espacial de 10 metros sobre a região da Lagoa dos Patos, Rio Grande do Sul, mostrando plantios de arroz irrigado em tons claros devido ao efeito de reflexão de canto decorrente da presença simultânea de hastes de arroz e lâmina de água. Tons mais escuros de cinza, adjacentes aos plantios de arroz, correspondem em sua maioria à vegetação herbácea, típica do bioma Pampa.

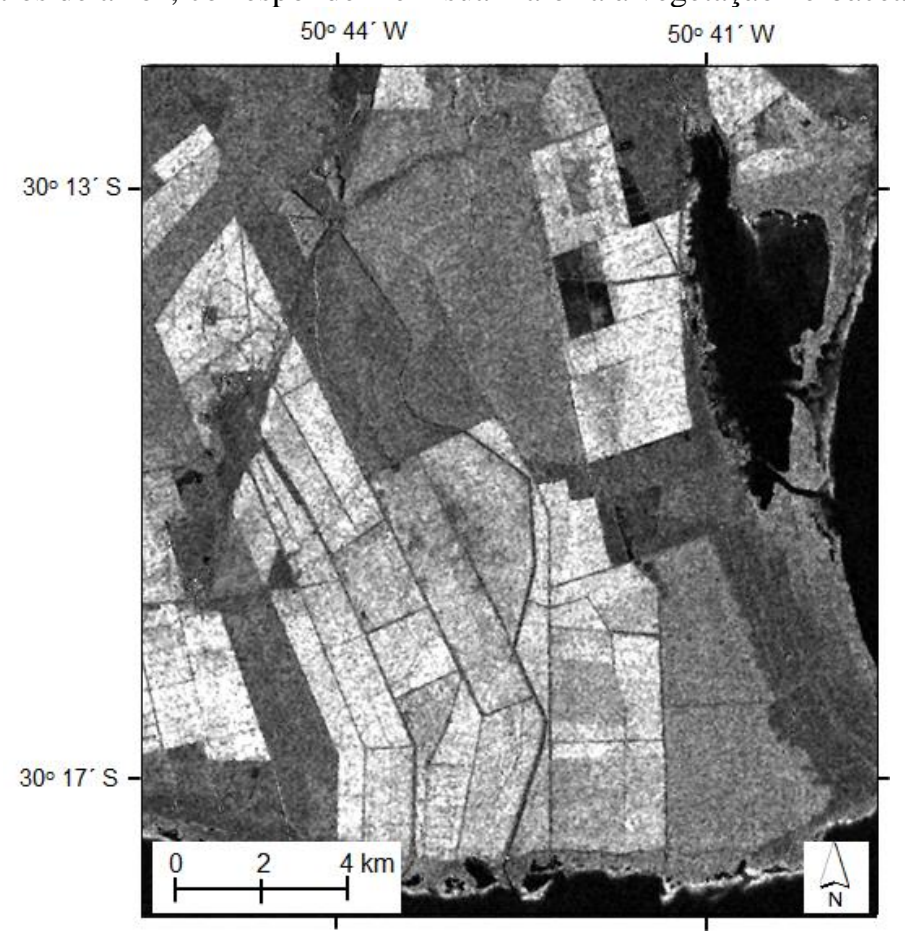

Fonte: Mucenic (2001).

\subsection{InSAR}

As aplicações iniciais do InSAR se concentraram principalmente no monitoramento dos deslocamentos de terra causados por terremotos. Uma das primeiras demonstrações práticas foi conduzida por Massonet et al. (1993), que utilizaram um par de dados SAR do satélite ERS-1 obtidos antes e depois do terremoto de Landers, Califórnia, ocorrido em 28 de junho de 1992, para caracterizar a deformação da superfície ao longo de um sistema de falha de $85 \mathrm{~km}$. O lançamento dos satélites Sentinel-1A e Sentinel-1B facilitou a produção de interferogramas, permitindo o monitoramento de terremotos sem a necessidade de dados fornecidos pela sismologia. Funning e Garcia (2019) estimaram, a partir de pesquisas bibliográficas, a existência de mais de 130 terremotos individuais monitorados usando dados InSAR. No entanto, a detecção da deformação do terreno com InSAR nem sempre é garantida, especialmente se ocorrer o fenômeno de decorrelação. A descorrelação corresponde à redução da correlação interferométrica, uma medida de coerência da fase interferométrica que pode ocorrer, por exemplo, dentro de certas condições de superfície, tais como vegetação densa ou topografia íngreme (FUNNING; GARCIA, 2019).

Mais recentemente, o leque de aplicações desta técnica foi ampliado principalmente para detecção de deslizamentos e subsistência de terras, com resolução inédita. Esses tipos de deformação do solo estão relacionados ao uso de águas subterrâneas para consumo humano, extração de materiais, por exemplo, atividades de mineração e extração de areias para construção civil, e ocupação humana de terrenos íngremes, entre outros. Atualmente, a combinação de dados de vários satélites SAR existentes permite o monitoramento quase que diário da deformação do solo causada por terremotos ou relacionadas com ações humanas. Conforme ressaltado por Biggs e Wright (2020), o InSAR está transformando o nosso entendimento sobre falhas, vulcões e estabilidade do terreno, contribuindo cada vez mais para aumentar a viabilidade das atividades de gerenciamento de desastres ambientais. 


\section{CONSIDERAÇÕES FINAIS}

O número relativamente elevado de satélites SAR lançados nos últimos anos e os planejados são bons indicadores de que a comunidade científica está convencida sobre o potencial do sensoriamento remoto por radar em diversos campos da ciência. $\mathrm{O}$ grande potencial das imagens de radar não se deve apenas à sua capacidade de geração de imagens em quase todas as condições climáticas, mas também porque as imagens SAR têm sido capazes de fornecer informações relevantes aos usuários em todo o mundo. Pelo menos duas aplicações são fortemente dependentes da tecnologia de radar, o monitoramento de derramamento de óleo usando a banda $\mathrm{C}$ e o desmatamento em países tropicais durante a estação chuvosa usando a banda $\mathrm{L}$. A disponibilidade da tecnologia TanDEM permitirá derivar MDEs com maior precisão e melhor resolução em comparação com os MDEs originalmente produzidos pela missão SRTM em 2000. A tomografia orbital bistática SAR possui grande potencial para se constituir em um excelente produto para monitorar o desmatamento nos trópicos e outras aplicações. $\mathrm{O}$ acesso gratuito a outros dados SAR adquiridos por sensores diferentes do Sentinel-1 são desejados para encorajar o uso mais intenso em diferentes aplicações e pesquisas. Como o processamento de imagens de radar não é uma tarefa fácil, produtos SAR prontos para utilização, como o índice de vegetação de radar (RVI), uma medição de micro-ondas bem estabelecida para estimativa da cobertura vegetal, também seriam úteis.

O uso de constelação de satélites de radar parece ser uma tendência irreversível em um futuro próximo, pois permite a redução dos intervalos de revisita, que têm sido um grande problema no sensoriamento remoto óptico. No entanto, os usuários de radar ainda estão ansiosos para ter a capacidade de polarização quádrupla instalada nos modos de aquisição operacionais. Essa capacidade é importante porque permite um processamento de imagem mais complexo, como a decomposição de imagens em três ou quatro componentes de diferentes mecanismos de espalhamento que não podem ser obtidos apenas por dados de amplitude. Dados de InSAR e interferometria diferencial por radar de abertura sintética (DInSAR), que utilizam informações de fase disponíveis em imagens complexas, permitirão o monitoramento de movimentos superficiais e deformações causados por terremotos e atividades vulcânicas com precisão de poucos milímetros. No entanto, a amplitude é o tipo mais básico de dados SAR; parece que seu uso ainda continuará por um tempo relativamente longo em várias aplicações, incluindo estimativa de biomassa florestal, monitoramento de desmatamento, previsão de safra, derramamento de óleo e detecção de embarcações, movimenta de geleiras e tráfego marítimo, entre outros.

Uma aplicação potencial da tecnologia SAR em um futuro próximo é o monitoramento do movimento de embarcações usando o satélite ALOS-4, com lançamento previsto para 2021. Este satélite será equipado com o Sistema de Identificação Automática (AIS) de embarcações. Assim, ao combinar os dados AIS com as imagens do ALOS-4, será possível identificar as embarcações cujos sistemas de rastreamento estiverem desligados durante a passagem do satélite. Outra aplicação potencial são os sensores SAR de altíssima resolução a bordo dos Veículos Aéreos Não-Tripulados (VANTs) operando no modo Spotlight (5-10 cm de resolução espacial) e em comprimentos de onda de micro-ondas curtos (por exemplo, banda $\mathrm{Ku}$ ) para reconhecimento de alvo ou até mesmo para estudos de detecção de mudança.

Algumas das questões desafiadoras que as pesquisas futuras em sensoriamento remoto por radar devem responder são:

a) Como as manchas escuras que frequentemente aparecem em imagens SAR relacionadas a derrames de óleo e manchas biogênicas presentes em superfícies oceânicas podem ser separadas sem a necessidade de inspeções locais ou de dados auxiliares?

b) Quando os sistemas SAR de próxima geração combinando alta resolução espacial (tipicamente, resolução de 1 metro) e alta periodicidade (tipicamente, 7 dias) estarão disponíveis, por exemplo, para melhorar a estimativa de umidade do solo e monitoramento de inundações e de safras agrícolas?

c) Uma das melhores formas de tornar os dados SAR populares e prontamente disponíveis para a observação global de processos dinâmicos na superfície da Terra é uma plataforma web semelhante ao Google Earth ${ }^{\mathrm{TM}}$, mas baseados em imagens SAR. Quando esse sonho se tornará realidade? 


\section{Contribuição dos Autores}

E. E. Sano contribuiu na conceptualização, curadoria dos dados, redação e revisão do texto. E. A. T. Matricardi e F. F. Camargo contribuíram na redação e revisão do texto.

\section{Conflitos de Interesse}

Os autores declaram que não há conflitos de interesse.

\section{Referências}

ALMEIDA-FILHO, R.; ROSENQVIST, A.; SHIMABUKURO, Y. E.; SANTOS, J. R. Evaluation and perspectives of using multitemporal L-band SAR data to monitor deforestation in the Brazilian Amazonia. IEEE Geoscience and Remote Sensing Letters, v. 2, n. 4, p. 409-412, 2005. DOI. 10.1109/LGRS.2005.856679

ALPERS, W.; HOLT, B.; ZENG, K. Oil spill detection by imaging radars: Challenges and pitfalls. Remote Sensing of Environment, v. 201, p. 133-147, 2017. DOI. 10.1016/j.rse.2017.09.002

ALSDORF, D. E.; SMITH, L. C.; MELACK, J. M. Amazon floodplain water level changes measured with interferometric SIR-C radar. IEEE Transactions on Geoscience and Remote Sensing, v. 39, n. 2, p. 423-431, 2001.

ALTESE, E.; BOLOGNANI, O.; MANCINI, M. Retrieving soil moisture over bare soil from ERS 1 synthetic aperture radar data: Sensitivity analysis based on a theoretical surface scattering model and field data. Water Resources Research, v. 32, p. 653-661, 1996. DOI. 10.1029/95WR03638

ALVES, S. A. S.; ASSANTE, L. R.; SANO, E. E.; MENESES, P. R. Abordagem metodológica baseada em imagens do SAR-R99B para identificar prováveis pistas de pouso não-homologadas na Amazônia. Acta Amazonica, v. 39, p. 723-726, 2009.

ARGENTI, F.; LAPINI, A.; ALAPARONE, L. A tutorial on speckle reduction in synthetic aperture radar images. IEEE Geoscience and Remote Sensing Magazine, v. 1, n. 3, p. 6-35, 2013. DOI. 10.1109/MGRS.2013.2277512

BAGHDADI, N.; HOLAH, N.; ZRIBI, M. Soil moisture estimation using multi-incidence and multipolarization ASAR data. International Journal of Remote Sensing, v. 27, p. 1907-1920, 2006. DOI. https://doi.org/10.1080/01431160500239032

BAGHDADI, N.; CHOKER, M.; ZRIBI, M.; EL HAJJ, M.; PALOSCIA, S.; VERHOEST, N. E. C.; LIEVENS, H.; BAUP, F.; MATTIA, F. A new empirical model for radar scattering from bare soil surfaces. Remote Sensing, v. 8, 920, 2016. DOI. 10.3390/rs8110920

BAN, Y.; ZHANG, P.; NASCETTI, A.; BEVINGTON, A. R.; WULDER, M. A. Near real-time wildfire progression monitoring with Sentinel-1 SAR time series and deep learning. Scientific Reports, v. 10, 1322, 2020. DOI. 10.1038/s41598-56967-x

BIGGS, J.; WRIGHT, T. J. How satellite InSAR has grown from opportunistic science to routine monitoring over the last decade. Nature Communications, v. 11, 3863, 2020. DOI. 10.1038/s41467-020-17587-6

CAMARGO, F. F.; SANO, E. E.; ALMEIDA, C. M.; MURA, J. C.; ALMEIDA, T. A comparative assessment of machine-learning techniques for land use and land cover classification of the Brazilian tropical savanna using ALOS-2/PALSAR-2 polarimetric images. Remote Sensing, v. 11, 1600, 2019. DOI. 10.3390/rs 11131600

CARrinO, T. A.; SILVA, A. M.; BOTELHO, N. F.; SILVA, A. A. C. Lógica fuzzy e técnica SAM para modelagem previsional do ouro no setor oeste da Província Mineral de Tapajós usando dados aerogeofísicos e de sensoriamento remoto. Revista Brasileira de Geofísica, v. 29, p. 535-554, 2011.

CANADA CENTRE FOR REMOTE SENSING (CCRS). Fundamentals of Remote Sensing. Disponível em: 
<https://www.nrcan.gc.ca/sites/www.nrcan.gc.ca/files/earthsciences/pdf/resource/tutor/fundam/pdf/fund amentals_e.pdf $>$. Acessado em: 16 out. 2020.

CIMINO, J.; ELACHI, C.; SETTLE, M. SIR-B - The second shuttle imaging radar experiment. IEEE Transactions on Geoscience and Remote Sensing, v. 24, n. 4, p. 445-452, 1986. DOI. 10.1109/TGRS.1986.289658

CLOUDE, S. R.; POTTIER, E. An entropy based classification scheme for land applications of polarimetric SAR. IEEE Transactions on Geoscience Remote Sensing, v. 35, n. 1, p. 68-78, 1997. DOI. $10.1109 / 36.551935$

COVEllo, F.; BATTAZZA, F.; COLETTA, A.; LOPINTO, E.; FIORENTINO, C.; PIETRANERA, L.; VALENTINI, G.; ZOFFOLI, S. COSMO-SkyMed an existing opportunity for observing the Earth. Journal of Geodynamics, v. 49, n. 3-4, p. 171-180, 2010. DOI. 10.1016/j.jog.2010.01.001

CANADIAN SPACE AGENCY (CSA). Technical features of the RCM. Disponível em: <https://www.asccsa.gc.ca/eng/satellites/radarsat/technical-features/default.asp>. Acessado em: 29 jul. 2020.

DECHESNE, C.; LEFÈVRE, S.; VALDAINE, R.; HAJDUCH, G.; FABLET, R. Ship identification and characterization in Sentinel-1 SAR images with multi-task deep learning. Remote Sensing, v. 11, n. 24, 2997, 2019. DOI. 10.3390/rs11242997

DOBSON, M. C.; PIERCE, L. E.; ULABY, F. T. Knowledge-based land-cover classification using ERS1/JERS-1 SAR composites. IEEE Transactions on Geoscience and Remote Sensing, v. 34, n. 1, p. 8399, 1996. DOI. 10.1109/36.481896

DYKSTRA, D. P. Reduced impact logging: concepts and issues. In: INTERNATIONAL CONFERENCE, 2001, Kuching, Malaysia. Proceedings... Bangkok: FAO, 2001. 526 p.

EL HAJJ, M.; BAGHDADI, N.; ZRIBI, M. Comparative analysis of the accuracy of surface soil moisture estimation from the C- and L-bands. International Journal of Applied Earth Observation and Geoinformation, v. 82, 101888, 2019. DOI. 10.1016/j.jag.2019.05.021

ENVI. Sentinel-1 Intensity Analysis in ENVI SARscape Tutorial. Disponível em: <https://www. .harrisgeospatial.com/portals/0/pdfs/Sentinel1 IntensityAnalysisTutorial.pdf>. Acessado em: 29 jul. 2020.

EUROPEAN SPACE AGENCY (ESA). Sentinel Application Platform (SNAP). SNAP 6.0 Help Guide. ESA, 2018.

EUROPEAN SPACE AGENCY (ESA). Sentinel-1 Toolbox. SAR Basics Tutorial. Paris: ESA, 2019, 19 p.

EUROPEAN SPACE AGENCY (ESA). SAOCOM. Disponível em: <https://directory.eoportal.org/web/eoportal/ satellite-missions/s/saocom>. Acessado em: 29 jul. 2020.

EVANS, D. L. Spaceborne imaging radar-C/X-band synthetic aperture radar (SIRC/X-SAR): a look back on the tenth anniversary. IEE Proceedings - Radar, Sonar and Navigation, v. 153, n. 2, p. 81-86, 2006. DOI. 10.1049/ip-rsn:20045095

EVANS, D. L.; ALPERS, W.; CAZENAVE, A.; ELACHI, C.; FARR, T.; GLACKIN, D.; HOLT, B.; JONES, L.; LIU, W. T.; McCANDLESS, W.; MENARD, Y.; MOORE, R.; NJOKU, E. Seasat - A 25-year legacy of success. Remote Sensing of Environment, v. 94, p. 384-404, 2005. DOI. 10.1016/j.rse.2004.09.011

FERRAZZOLI, P.; PALOSCIA, S.; PAMPALONI, P.; SCHIAVON, G.; SIGISMONDI, S.; SOLIMINI, D. The potential of multifrequency polarimetric SAR in assessing agricultural and arboreous biomass. IEEE Transactions on Geoscience and Remote Sensing, v. 35, n. 1, p. 5-17, 1997. DOI. 10.1109/36.551929

FOX, P. A.; LUSCOMBE, A. P.; THOMPSON, A. A. RADARSAT-2 SAR modes development and utilization. Canadian Journal of Remote Sensing, v. 30, n. 3, p. 258-264, 2004. DOI. 10.5589/m04-014

FREEMAN, A.; DURDEN, S. L. A three-component scattering model for polarimetric SAR Data. IEEE Transactions on Geoscience and Remote Sensing, v. 36, n. 3, p. 963-973, 1998. DOI. $10.1109 / 36.673687$

FUNNING, G. J.; GARCIA, A. A systematic study of earthquake detectability using Sentinel-1Interferometric Wide-Swath data. Geophysical Journal International, v. 216, p. 332-349, 2019. DOI. 


\subsection{3/gji/ggy426}

GUERRA, J. B.; MURA, J. C.; FREITAS, C. C. Discriminação de incrementos de desflorestamento na Amazônia com dados SAR R99B em banda L. Acta Amazonica, v. 40, p. 557-566, 2010.

HAGENSIEKER, R.; WASKE, B. Evaluation of multi-frequency SAR images for tropical land cover mapping. Remote Sensing, v. 10, 257, 2018. DOI. 10.3390/rs10020257

HANSSEN, R. F. Radar Interferometry: Data Interpretation and Error Analysis. New York: Kluwer Academic Publishers, 2002, 308 p.

HELLMANN, M. P. SAR polarimetry tutorial. Rennes: University of Rennes, 2001. 109 p. Disponível em: <http://epsilon.nought.de/>. Acessado em: 02 ago. 2020.

HENDERSON, F. M.; LEWIS, A. J. Principles \& Applications of Imaging Radar. Manual of Remote Sensing. Nova York: John Wiley \& Sons, $3^{\text {rd }}$ ed., v. 2, 1998.

HESS, L. L.; MELACK, J. M.; FILOSO, S.; WANG, Y. Delineation of inundated area and vegetation along the Amazon floodplain with SIR-C synthetic aperture radar. IEEE Transactions on Geoscience and Remote Sensing, v. 33, n. 4, p. 896-903, 1995. DOI. 10.1109/36.406675

HUBER, S.; ALMEIDA, F. Q.; VILLANO, M.; YOUNIS, M.; KRIEGER, G.; MOREIRA, A. Tandem-L: A technical perspective on future spaceborne SAR sensors for Earth observation. IEEE Transactions on Geoscience and Remote Sensing, v. 56, n. 8, p. 4792-4807, 2018. DOI. 10.1109/TGRS.2018.2837673

INSTITUTO BRASILEIRO DE GEOGRAFIA E ESTATÍSTICA (IBGE). Biblioteca. Catálogo. Rio de Janeiro: IBGE. Disponível em: <https://biblioteca.ibge.gov.br/index.php/bibliotecacatalogo $?$ acervo $=$ todos $\&$ campo $=$ todos $\&$ notqry $=\&$ opeqry $=\&$ texto $=$ RADAMBRASIL $\&$ digital $=$ false $\&$ fra seexata=>. Acessado em: 29 jul. 2020.

ISHITSUKA, N. Identification of paddy rice areas using SAR: Some case studies in Japan. Japan Agricultural Research Quarterly, v. 52, n. 3, p. 197-204, 2018. DOI. 10.6090/jarq.52.197

IVANOV, Y. Y. Assessment of marine oil pollution using Kosmos-1980 and Almaz-1 radar images. Mapping Sciences and Remote Sensing, v. 35, n. 3, p. 202-217, 1998. DOI. 10.1080/07493878.1998.10642092

JACKSON, C. M.; ADAM, E. Remote sensing of selective logging in tropical forests: Current state and future directions. iForest - Biogeosciences and Forestry, v. 13, p. 286-300, 2020. DOI. 10.3832/ifor3301-013

JAXA. Japan Aerospace Exploration Agency. ALOS-2 basic observation scenario ( $2^{\mathrm{a}}$ ed. rev.A).Tsukuba: JAXA, 2017, 35 p.

JENSEN, J. R. Remote Sensing of the Environment. An Earth Resources Perspective. Upper Saddle River: Prentice Hall Inc., 2000.

KASISCHKE, E. S.; MELACK, J. M.; DOBSON, M. C. The use of imaging radars for ecological applications - a review. Remote Sensing of Environment, v. 59, n. 2, p. 141-156, 1997. DOI. 10.1016/S00344257(96)00148-4

KOYAMA, C. N.; WATANABE, M.; HAYASHI, M.; OGAWA, T.; SHIMADA, M. Mapping the spatialtemporal variability of tropical forests by ALOS-2 L-band SAR big data analysis. Remote Sensing of Environment, v. 233, 111372, 2019. DOI. 10.1016/j.rse.2019.111372

KRIEGER, G.; MOREIRA, A.; FIEDLER, H.; HAJNSEK, I.; WERNER, M.; YOUNIS, M.; ZINK, M. TanDEM-X: A satellite formation for high-resolution SAR interferometry. IEEE Transactions on Geoscience and Remote Sensing, v. 45, n. 11, p. 3317-3341, 2007. DOI. 10.1109/TGRS.2007.900693

KRIEGER, G.; ZINK, M.; BACHMANN, M.; BRÄUTIGAM, B.; SCHULZE, D.; MARTONE, M.; RIZZOLI, P.; STEINBRECHER, U.; ANTONY, J. W.; DE ZAN, F.; HAJNSEK, I.; PAPATHANASSIOU, K.; KUGLER, F.; CASSOLA, M. R.; YOUNIS, M.; BAUMGARTNER, S.; LÓPEZ-DEKKER, P.; PRATS, P.; MOREIRA, A. TanDEM-X: A radar interferometer with two formation-flying satellites. Acta Astronautica, v. 89, p. 83-98, 2013. DOI. 10.1016/j.actaastro.2013.03.008

KUROSU, T.; FUJITA, M.; CHIBA, K. Monitoring of rice crop growth from space using the ERS-1 C-band 
SAR. IEEE Transactions on Geoscience and Remote Sensing, v. 33, n. 4, p. 1092-1096, 1995. DOI. $10.1109 / 36.406698$

LA ROSA, L. E. C.; FEITOSA, R. Q.; HAPP, P. N.; SANCHES, I. D.; COSTA, G. A. O. P. Combining deep learning and prior knowledge for crop mapping in tropical regions from multitemporal SAR image sequences. Remote Sensing, v. 11, 2029, 2019. DOI. 10.3390/rs11172029

LAVROVA, O. Y.; KOSTIANOY, A. G. Catastrophic oil spill in the Gulf of Mexico in April-May 2010. Izvestiya, Atmospheric and Oceanic Physics, v. 47, n. 9, p. 1114-1118, 2011. DOI. 10.1134/S0001433811090088

LE TOAN, T.; RIBBES, F.; WANG, L. F.; FLOURY, N.; DING, K. H.; KONG, J. A.; FUJITA, M. Rice crop mapping and monitoring using ERS-1 data based on experiment and modeling results. IEEE Transactions on Geoscience and Remote Sensing, v. 35, n. 1, p. 41-56, 1997. DOI. 10.1109/36.551933

LEE, J.; POTTIER, E. Polarimetric Radar Imaging: from Basics to Applications. New York: CRC Press, 2009, $398 \mathrm{p}$.

LEIFER, I.; LEHR, W. J.; SIMECEK-BEATTY, D.; BRADLEY, E.; CLARK, R.; DENNISON, P.; HU, Y.; MATHESON, S.; JONES, C. E.; HOLT, B.; REIF, M.; ROBERTS, D. A.; SVEJKOVSKY, J.; SWAYZE, G.; WOZENCRAFT, J. State of the art satellite and airborne marine oil spill remote sensing: Application to the BP Deepwater Horizon oil spill. Remote Sensing of Environment, v. 124, p. 185-209, 2012. DOI. 10.1016/j.rse.2012.03.024

LI, H.; HE, Y.; WANG, W. Improving ship detection with polarimetric SAR based on convolution between co-polarization channels. Sensors, v. 9, n. 2, p. 1221-1236, 2009. DOI. 10.3390/s90201221

LIMA, M. I. C. Projeto Radam: Uma Saga Amazônica. Belém: Paka-Tatu, 2014.

LU, J.; LI, X. Review of rice-fish-farming systems in China - One of the Globally Important Ingenious Agricultural Heritage Systems (GIAHS). Aquaculture, v. 260, n. 1-4, p. 106-113, 2006. DOI. 10.1016/j.aquaculture.2006.05.059

MANDAL, D.; KUMAR, V.; RATHA, D.; DEY, S.; BHATTACHARYA, A.; LOPEZ-SANCHEZ, J. M.; McNAIRN, H.; RAO, Y. S. Dual polarimetric radar vegetation index for crop growth monitoring using Sentinel-1 SAR data. Remote Sensing of Environment, v. 247, 111954, 2020. DOI. 10.1016/j.rse.2020.111954

MARTONE, M.; RIZZOLI, P.; WECKLICH, C.; GONZÁLEZ, C.; BUESO-BELLO, J. L.; VALDO, P.; SCHULZE, D.; ZINK, M.; KRIEGER, G.; MOREIRA, A. The global forest/non-forest map from TanDEM-X interferometric SAR data. Remote Sensing of Environment, v. 205, p. 352-373, 2018. DOI. 10.1016/j.rse.2017.12.002

MASSONNET, D.; ROSSI, M.; CARMONA, C.; ADRAGNA, F.; PELTZER, G.; FEIGL, K.; RABAUTE, T. The displacement field of the Landers earthquake mapped by radar interferometry. Nature, v. 364, 6433, p. $138-142$. DOI. $10.1038 / 364138 \mathrm{a} 0$

MATRICARDI, E. A. T.; SKOLE, D. L.; PEDLOWSKI, M. A.; CHOMENTOWSKI, W. Assessment of forest disturbances by selective logging and forest fires in the Brazilian Amazon using Landsat data. International Journal of Remote Sensing, v. 34, p. 1057-1086, 2013. DOI: 10.1080/01431161.2012.717182

MIGLIACCIO, M.; NUNZIATA, F.; MONTUORI, A.; PAES, R. L. Single-look complex COSMO-SkyMed SAR data to observe metallic targets at sea. IEEE Journal of Selected Topics in Applied Earth Observations and Remote Sensing, v. 5, n. 3, p. 893-901, 2012. DOI. 10.1109/JSTARS.2012.2184271

MONTEIRO, A. L. S.; SOUZA Jr., C. M.; BARRETO, P. G.; PANTOJA, F. L. S.; GERWING, J. J. Impactos da exploração madeireira e do fogo em florestas de transição da Amazônia Legal. Scientia Forestalis, n. 65, p. 11-21, 2004.

MOREIRA, A.; KRIEGER, G.; HAJNSEK, I.; PAPATHANASSIOU, K.; YOUNIS, M.; LOPEZ-DEKKER, P.; HUBER, S.; VILLANO, M.; PARDINI, M.; EINEDER, M.; DE ZAN, F.; PARIZZI, A. Tandem-L: A highly innovative bistatic SAR mission for global observation of dynamics processes on the Earth's 
surface. IEEE Geoscience and Remote Sensing Magazine, v. 3, n. 2, p. 8-23, 2015. DOI. 10.1109/mgrs.2015.2437353

MUCENIC, N. Uso do radar (banda C) no mapeamento de arroz irrigado no estado do Rio Grande do Sul (dissertado de mestrado em Sensoriamento Remoto), Porto Alegre: Universidade Federal do Rio Grande do Sul, 2001.

MURA, J. C.; PARADELLA, W. R.; DUTRA, L. V.; SANTOS, J. R.; RUDORFF, B. F. T.; MIRANDA, F. P.; SILVA, M. M. Q.; SILVA, W. F. MAPSAR image simulation based on L-band polarimetric data from the SAR-R99B airborne sensor (SIVAM system). Sensors, v. 9, p. 102-117, 2009. DOI. $10.3390 / \mathrm{s} 90100102$

NANNINI, M.; MARTONE, M.; RIZZOLI, P.; PRATS-IRAOLA, P.; RODRIGUEZ-CASSOLA, M.; REIGBER, A.; MOREIRA, A. Coherence-based SAR tomography for spaceborne applications. Remote Sensing of Environment, v. 225, p. 107-114, 2019. DOI. 10.1016/j.rse.2019.01.040

NURTYANWAN, R.; FERDIYANTI, G. A.; BUDIHARTO, A.; WIKANTIKA, K. Modelling of surface roughness on agriculture area using Radarsat-2 satellite. IOP Conference Series: Earth and Environmental Science, v. 500, 012080, 2020. DOI. 10.1088/1755-1315/500/1/012080

OUCHI, K. Recent trend and advance of synthetic aperture radar with selected topics. Remote Sensing, v. 5, p. 716-807, 2013. DOI. 10.3390/rs5020716

PARADELLA, W. R.; SANTOS, A. R.; VENEZIANI, P.; CUNHA, E. S. P. Radares imageadores nas geociências: Estado da arte e perspectivas. Revista Brasileira de Cartografia, v. 57, n. 1, p. 56-62, 2005.

PEREIRA, L. O.; FREITAS, C. C.; SANT'ANNA, S. J. S.; REIS, M. S. ALOS/PALSAR data evaluation for land use and land cover mapping in the Amazon region. IEEE Journal of Selected Topics in Applied Earth Observations and Remote Sensing, v. 9, n. 12, p. 5413-5423, 2016. DOI. 10.1109/JSTARS.2016.2622481

PINTO, W. H. A.; LUCHIARI, A. Uso das imagens SAR R99B para mapeamento geomorfológico do canal do Ariaú no município de Iranduba-AM. Revista de Geografia, v. 34, p. 209-229, 2017.

PITZ, W.; MILLER, D. The TerraSAR-X satellite. IEEE Transactions on Geoscience and Remote Sensing, v. 48, n. 2, p. 615-622, 2010. DOI. 10.1109/TGRS.2009.2037432

PULELlA, A.; SANTOS, R. A.; SICA, F.; POSOVSZKY, P.; RIZZOLI, P. Multi-temporal Sentinel-1 backscatter and coherence for rainfall mapping. Remote Sensing, 12, 847, 2020. DOI. $10.3390 /$ rs 12050847

REICH, J.; VERBESSELT, J.; HOEKMAN, D.; HEROLD, M. Fusing Landsat and SAR time series to detect deforestation in the tropics. Remote Sensing of Environment, v. 156, p. 276-293, 2015. DOI. 10.1016/j.rse.2014.10.001

RICHARDS, J. A. Remote Sensing with Imaging Radar. Berlin: Springer, 2009. 361 p.

RIZZOLI, P.; MARTONE, M.; GONZALEZ, C.; WECKLICH, C.; TRIDON, D. B.; BRAUTIGAM, B.; BACHMANN, M.; SCHULZE, D.; FRITZ, T.; HUBER, M.; WESSEL, B.; KRIEGER, G.; ZINK, M.; MOREIRA, A. Generation and performance assessment of the global TanDEM-X digital elevation model. ISPRS Journal of Photogrammetry and Remote Sensing, v. 132, p. 119-139, 2017. DOI. 10.1016/j.isprsjprs.2017.08.008

ROSENQVIST, A.; SHIMADA, M.; LUCAS, R.; CHAPMAN, B.; PAILLOU, P.; HESS, L.; LOWRY, J. The Kyoto \& Carbon Initiative - a brief summary. IEEE Journal of Selected Topics in Applied Earth Observations and Remote Sensing, v. 3, n. 4, p. 551-553, 2010. DOI. 10.1109/JSTARS.2010.2086270

ROSENQVIST, A.; SHIMADA, M.; SUZUKI, S.; OHGUSHI, F.; TADONO, T.; WATANABE, M.; TSUZUKU, K.; WATANABE, T.; KAMIJO, S.; AOKI, E. Operational performance of the ALOS global systematic acquisition strategy and observation plans for ALOS-2 PALSAR-2. Remote Sensing of Environment, v. 155, p. 3-12, 2014. DOI. 10.1016/j.rse.2014.04.011

SAATCHI, S. S.; SOARES, J. V.; ALVES, D. S. Mapping deforestation and land use in Amazon rainforest 
by using SIR-C imagery. Remote Sensing of Environment, v. 59, p. 191-202, 1997. DOI. 10.1016/S0034-4257(96)00153-8

SANO, E. E.; FERREIRA, L. G.; HUETE, A. R. Synthetic aperture radar (L-band) and optical vegetation indices for discriminating the Brazilian savanna physiognomies: a comparative analysis. Earth Interactions, v. 9, 15, 2005. DOI. 10.1175/EI117.1

SANO, E. E.; MENESES, P. R.; ALMEIDA, T. Reflectância dos alvos na faixa de micro-ondas. In: MENESES, P. R.; ALMEIDA, T.; BAPTISTA, G. M. M. (Orgs.). Reflectância dos Materiais Terrestres. Análise e Interpretação. São Paulo: Oficina de Textos, Cap. 10, 2019. p. 291-315.

SANO, E. E.; HUETE, A. R.; TROUFLEAU, D.; MORAN, M. S.; VIDAL, A. Relation between ERS-1 synthetic aperture radar data and measurements of surface roughness and moisture content of rocky soils in a semiarid rangeland. Water Resources Research, v. 34, n. 6, p. 1491-1498, 1998a. DOI. 10.1029/98WR00032

SANO, E. E.; MORAN, M. S.; HUETE, A. R.; MIURA, T. C- and multiangle Ku-band synthetic aperture radar data for bare soil moisture estimation in agricultural areas. Remote Sensing of Environment, v. 64, p. 77-90, 1998b. DOI. 10.1016/S0034-4257(97)00170-3

SARMAP. SARSCAPE. Caslano: SARMAP, 2020. Disponível em: <http://www.sarmap.ch/wp/index.php/software/sarscape/>. Acessado em: 3 ago. 2020.

SCHLUND, M.; ERASMI, S. Sentinel-1 time series data for monitoring the phenology of winter wheat. Remote Sensing of Environment, v. 246, 111814, 2020. DOI. 10.1016/j.rse.2020.111814

SILVA, A. Q.; PARADELLA, W. R.; FREITAS, C. C.; OLIVEIRA, C. G. Evaluation of digital classification of polarimetric SAR data for iron-mineralized laterites mapping in the Amazon region. Remote Sensing, v. 5, p. 3101-3122, 2013. DOI. 10.3390/rs5063101

SILVA, V. S.; SANO, E. E.; ALMEIDA, T.; MESQUITA JÚNIOR, H. N. Discriminação de classes de cobertura vegetal em uma região de transição Amazônia/Cerrado no estado de Mato Grosso por meio de imagens do satélite ALOS-2/PALSAR-2. Revista Brasileira de Cartografia, 2020, no prelo.

SINGHA, M.; DONG, J.; ZHANG, G.; XIAO, X. High resolution paddy rice maps in cloud-prone Bangladesh and Northeast India using Sentinel-1 data. Scientific Data, v. 6, 2019. DOI.: 10.1038/s41597-019-0036-3

SOUZA-FILHO, P. W. M.; PARADELLA, W. R.; RODRIGUES, S. W. P.; COSTA, F. R.; MURA, J. C.; GONÇALVES, F. D. Discrimination of coastal wetland environments in the Amazon region based on multi-polarized L-band airborne synthetic aperture radar imagery. Estuarine, Coastal and Shelf Science, v. 95, p. 88-98, 2011. DOI. 10.1016/j.ecss.2011.08.011

SRIVASTAVA, H. S.; PATEL, P.; MANCHANDA, M. L.; ADIGA, S. Use of multi-incidence angle RADARSAT-1 SAR data to incorporate the effect of surface roughness in soil moisture estimation. IEEE Transactions on Geoscience and Remote Sensing, v. 41, n. 7, p. 1638-1640, 2003. DOI. 10.1109/TGRS.2003.813356

STEINMETZ, S.; BRAGA, H. J. Zoneamento de arroz irrigado por épocas de semeadura nos estados do Rio Grande do Sul e de Santa Catarina. Revista Brasileira de Agrometeorologia, v. 9, n. 3, p. 429-438, 2001.

STONE, T. A.; WOODWELL, G. M. Shuttle imaging radar. An analysis of land use in Amazonia. International Journal of Remote Sensing, v. 9, n. 1, p. 95-105, 1988 . DOI. 10.1080/01431168808954839

TAKAKU, J.; TADONO, T.; TSUTSUI, K. Generation of high resolution global DSM from ALOS PRISM. The International Archives of the Photogrammetry, Remote Sensing and Spatial Information Sciences, v. XL-4, p. 243-248, 2014. DOI. 10.5194/isprsarchives-XL-4-243-2014

THOMPSON, I. D.; GUARIGUATA, M. R.; OKABE, K.; BAHAMONDEZ, C.; NASI, R.; HEYMELL, V.; SABOGAL, C. An operational framework for defining and monitoring forest degradation. Ecology and Society, v. 18, n. 2, 20, 2013. DOI. 10.5751/ES-05443-180220

TORRES, R.; SNOEIJ, P.; GEUDTNER, D.; BIBBY, D.; DAVIDSON, M.; ATTEMA, E.; POTIN, P.; 
ROMMEN, B.; FLOURY, N.; BROWN, M.; TRAVER, I. N.; DEGHAYE, P.; DUESMANN, B.; ROSICH, B.; MIRANDA, N.; BRUNO, C.; L’ABBATE, M.; CROCI, R.; PIETROPAOLO, A.; HUCHLER, M.; ROSTAN, F. GMES Sentinel-1 mission. Remote Sensing of Environment, v. 120, p. 9-24, 2012. DOI. 10.1016/j.rse.2011.05.028

TRITSCH, I.; SIST, P.; NARVAES, I. S.; FREITAS, L. J. M.; BLANC, L.; BOURGOIN, C.; CORNU, G.; GOND, V. Multiple patterns of forest disturbance and logging shape forest landscape in Paragominas, Brasil. Forests, v. 7, 315, 2016. DOI: 10.3390/f7120315

ULABY, F. T.; BATLIVALA, P. P. Optimum radar parameters for mapping soil moisture. IEEE Transactions on Geoscience Electronics, v. 14, n. 2, p. 81-93, 1976. DOI. 10.1109/TGE.1976.294414

ULABY, F. T.; DUBOIS, P. C.; VAN ZYL, J. Radar mapping of surface soil moisture. Journal of Hydrology, v. 184, p. 57-84, 1996. DOI. 10.1016/0022-1694(95)02968-0

VALENZUELA, G. R. Theories for the interaction of electromagnetic and oceanic waves - a review. Boundary-Layer Meteorology, v. 13, n. 1-4, p. 61-85, 1978. DOI. 10.1007/BF00913863

VAN DER SANDEN, J. J. Radar remote sensing to support tropical forest management (tese de doutorado em Agronomia), 1997, Wageningen: Wageningen Agricultural University, 1997, 330 p.

WATANABE, M.; KOYAMA, C. N.; HAYASHI, M.; NAGATANI, I.; SHIMADA, M. Early-stage deforestation detection in the tropics with L-band SAR. IEEE Journal of Selected Topics in Applied Earth Observations and Remote Sensing, v. 11, n. 6, p. 2127-2133, 2018. DOI. 10.1109/JSTARS.2018.2810857

WU, F.; WANG, C.; ZHANG, H.; ZHANG, B.; TANG, Y. Rice crop monitoring in South China with RADARSAT-2 quad-polarization SAR data. IEEE Geoscience and Remote Sensing Letters, v. 8, n. 2, 2011. DOI. 10.1109/LGRS.2010.2055830

WU, X.; LIU, L.; GUO, X.; LIU, Z.; LI, K.; SHAO, Y. Comparison of water cloud models with different layers for rice yield estimation from a single TerraSAR image. Remote Sensing Letters, v. 11, n. 9, p. 876-882, 2020. DOI. 10.1080/2150704X.2020.1781284

YAMAGUCHI, Y.; MORIYAMA, T.; ISHIDO, M.; YAMADA, H. Four-component scattering model for polarimetric SAR image decomposition. IEEE Transactions on Geoscience Remote Sensing, v. 43, n. 8, p. 1699-1706, 2005. DOI. 10.1109/TGRS.2005.852084

YANG, S.; ZHAO, X.; LI, B.; HUA, G. Interpreting RADARSAT-2 quad-polarization SAR signatures from rice paddy based on experiments. IEEE Geoscience and Remote Sensing Letters, v. 9, n. 1, p. 65-69, 2012. DOI. 10.1109/LGRS.2011.2160613

ZHANG, M.; CHEN, F.; LIANG, D.; TIAN, B.; YANG, A. Use of Sentinel-1 GRD SAR images to delineate flood extent in Pakistan. Sustainability, v. 12, 5784, 2020. DOI. 10.3390/su12145784

ZINK, M.; BACHMANN, M.; BRÄUTIGAM, B.; FRITZ, T.; HAJNSEK, I.; KRIEGER, G.; MOREIRA, A.; WESSEL, B. TanDEM-X: The new global DEM takes shape. IEEE Geoscience and Remote Sensing Magazine, v. 2, n. 2, p. 8-23, 2014. DOI. 10.1109/mgrs.2014.2318895

\section{Biografia dos autores}

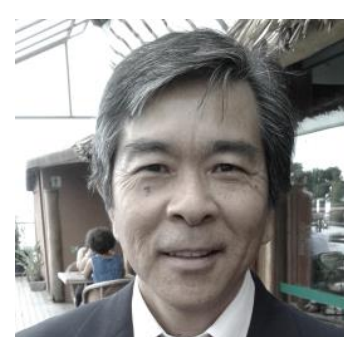

Edson Eyji Sano, Echaporã/SP - Brasil, 1958. Geólogo formado pela Universidade de São Paulo (USP), São Paulo/SP, mestre em Sensoriamento Remoto pelo Instituto Nacional de Pesquisas Espaciais (INPE), São José dos Campos/SP, e Ph.D. em Ciência do Solo pela Universidade do Arizona, Tucson/Arizona. Pesquisador da Embrapa Cerrados desde 1989. Seu interesse de pesquisa é na área de monitoramento agrícola e ambiental por meio de dados de sensoriamento remoto óptico e de radar, principalmente nos biomas Cerrado e Amazônia. 

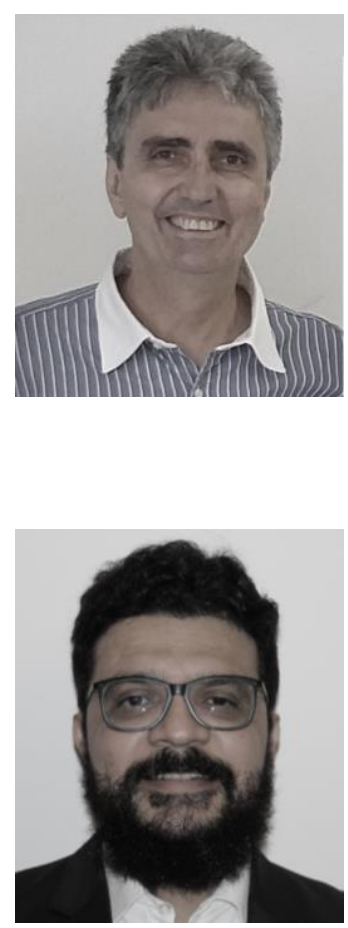

Eraldo Aparecido Trondoli Matricardi, Presidente Prudente/SP, Brasil, 1963. Engenheiro Florestal formado pela Universidade Federal de Mato Grosso (UFMT), Cuiabá/MT, especialista em Sensoriamento Remoto pela Universidade Federal de Santa Maria (UFSM), Santa Maria/RS, mestre e doutor em Geografia pela Michigan State University (MSU), USA. Professor Associado na Universidade de Brasília (UnB), com início em 2009. Seu interesse de pesquisa é na área de degradação florestal, mudanças de uso e cobertura da terra usando dados de sensoriamento remoto, mudanças climáticas e análises de paisagens.

Flávio Fortes Camargo, Curitiba/PR - Brasil, 1978. Geógrafo formado pela Universidade de São Paulo (USP), São Paulo/SP, mestre em Sensoriamento Remoto pelo Instituto Nacional de Pesquisas Espaciais (INPE), São José dos Campos/SP, e Ph.D. em Geociências Aplicadas e Geodinâmica pela Universidade de Brasília (IG-UnB), Brasília/DF. Analista em Infraestrutura de Transportes do DNIT desde 2013. Seu interesse de pesquisa é na área de geomorfologia e monitoramento do uso e cobertura do solo por meio de dados de sensoriamento remoto óptico, radar e de técnicas de inteligência artificial.

Esta obra está licenciada com uma Licença Creative Commons Atribuição 4.0 Internacional - CC BY.Esta licença permite que outros distribuam, remixem, adaptem e criem a partir do seu trabalho, mesmo para fins comerciais, desde que lhe atribuam o devido crédito pela criação original. 